\title{
Transfer matrix spectrum for cyclic representations of the 6-vertex reflection algebra I
}

\author{
Jean Michel Maillet, Giuliano Niccoli and Baptiste Pezelier \\ University of Lyon, ENS de Lyon, University Claude Bernard Lyon 1, CNRS, Laboratoire de \\ Physique, UMR 5672, F-69342 Lyon, France \\ maillet@ens-lyon.fr, giuliano.niccoli@ens-lyon.fr, baptiste.pezelier@ens-lyon.fr
}

\begin{abstract}
We study the transfer matrix spectral problem for the cyclic representations of the trigonometric 6-vertex reflection algebra associated to the Bazanov-Stroganov Lax operator. The results apply as well to the spectral analysis of the lattice sine-Gordon model with integrable open boundary conditions. This spectral analysis is developed by implementing the method of separation of variables (SoV). The transfer matrix spectrum (both eigenvalues and eigenstates) is completely characterized in terms of the set of solutions to a discrete system of polynomial equations in a given class of functions. Moreover, we prove an equivalent characterization as the set of solutions to a Baxter's like T-Q functional equation and rewrite the transfer matrix eigenstates in an algebraic Bethe ansatz form. In order to explain our method in a simple case, the present paper is restricted to representations containing one constraint on the boundary parameters and on the parameters of the Bazanov-Stroganov Lax operator. In a next article, some more technical tools (like Baxter's gauge transformations) will be introduced to extend our approach to general integrable boundary conditions.
\end{abstract}

(c) (1) Copyright J. M. Maillet et al.

This work is licensed under the Creative Commons

Attribution 4.0 International License.

Published by the SciPost Foundation.
Received 11-11-2016

Accepted 24-02-2017

Published 28-02-2017

doi:10.21468/SciPostPhys.2.1.009

\section{Contents}

1 Introduction $\quad 2$

2 Cyclic representations of the 6-vertex Yang-Baxter algebra 4

2.1 Bulk transfer matrix and quantum determinant 5

3 Cyclic representations of the 6-vertex reflection algebra $\quad 6$

$\begin{array}{lll}3.1 & \text { Boundary transfer matrix and quantum determinant } & 7\end{array}$

4 SoV representation of cyclic 6-vertex reflection algebra $\quad 8$

4.1 Pseudo-vacuum states 8

4.2 Representation of the reflection algebra in $\mathscr{B}_{-}(\lambda)$-eigenstates basis 9

4.3 Change of basis and SoV spectral decomposition of the identity 13

$\begin{array}{lll}4.4 & \text { Separate states and their scalar products } & 14\end{array}$ 
6 Functional equation characterizing the $\mathscr{T}$-spectrum 20

$\begin{array}{llr}7 & \text { Conclusions } & 26\end{array}$

A Appendix $\quad 26$

A.1 General proof of diagonalizability of $\mathscr{B}_{-}(\lambda) \quad 26$

A.2 The lattice sine-Gordon model with integrable boundaries 28

$\begin{array}{lll}\text { A.3 Reduction to inhomogeneous chiral Potts representations } & 30\end{array}$

A.4 Reduction to the XXZ spin $s$ open chains at the $p=2 s+1$ roots of unit 31

References

\section{Introduction}

The study of quantum models with integrable open boundary conditions has attracted a large research interest, e.g. see [1-52] and references therein. These models are of physical interest as they can describe both equilibrium and out of equilibrium physics; e.g. some interesting applications concern the description of classical stochastic relaxation processes, like ASEP [53, 54], [48-51], and quantum transport properties in spin systems $[55,56]$.

In this paper we start the analysis of the class of open integrable quantum models associated to cyclic representations [57-61] of the 6-vertex reflection algebra. The literature of these models is so far rather sparse with the exception of some rather special representations and boundary conditions, like the open XXZ chains at the roots of unity, that can be traced back to these representations under some special constraints, and for which some results are known in the framework of algebraic Bethe ansatz (ABA) $[52,62,63]$, fusion of transfer matrices and truncation identities $[6,7,64-67]$.

In order to study the general representations and boundary conditions we have to go beyond traditional methods [62-83] which do not apply for these general settings. This is done by developing the Sklyanin's SoV method [84-87] for this class of models, a method that has the advantage to lead (mainly by construction) to the complete characterization of the spectrum (eigenvalues and eigenvectors) and has proven to be applicable for a large variety of integrable quantum models [28-36, 88-102], where traditional methods fail. Moreover, the SoV approach has the advantage to allow also for the study of the dynamics of the models as it leads to universal determinant formulae for matrix elements of local operators on transfer matrix eigenstates as shown for different classes of models, first in [97], and then in many other cases in $[33,88,89,99,100,103]$. Moreover, the analysis developed in $[104,105]$ makes it possible to compute homogeneous and then thermodynamic limit of these matrix elements opening the way to the computation of the corresponding correlation functions.

Let us recall that in [3], Sklyanin has shown how to construct classes of quantum models with integrable boundaries in the framework of the so-called Quantum Inverse Scattering Method [62-79], constructing in particular associated families of commuting transfer matrices (conserved charges of the model). In fact, Sklyanin's construction allows to use solutions of the Yang-Baxter equation to generate new solutions of the reflection equation [4] once a scalar solution of this last equation is known. Then as a consequence of the reflection equation these new solutions generate commuting transfer matrices [3]. 
Sklyanin has used the 6-vertex case and the associated XXZ spin 1/2 quantum chains [80, 106-108] to develop an explicit example of this construction. However as pointed out in [3] similar constructions applies also to other 6-vertex cases like the non-linear Schrödinger and the Toda chains as well as for models associated to the 8 -vertex case like the XYZ spin $1 / 2$ quantum chains. Further integrable quantum models with open boundary conditions have been presented following the Sklyanin's construction. Interesting examples are the higher spin open quantum chains $[17,18]$, the higher rank open quantum spin chains [40-42] and the Hubbard model [109-112] with integrable open boundaries [43-48]. In fact, this mainly results in the possibility to associate to any closed integrable quantum model (characterized by a solution of the Yang-Baxter equation) new open integrable quantum models (characterized by the associated Sklyanin's solutions of the reflection equation).

Here, we characterize the spectral problem and we start the analysis of the dynamical problem (by determining scalar products of separate states) for the class of models in the Sklyanin's construction associated to general scalar solutions of the 6 -vertex reflection equation $[11,13]$ and the general Bazhanov-Stroganov cyclic solution of the 6-vertex Yang-Baxter algebra [113].

It may be instructive to recall some main literature on open integrable quantum chains, even for different representations with regards to those studied here. Indeed, this allows us to point out the difficulties that arise in their analysis and that we have also encountered for the models studied in this paper and to give the motivations for the approach that we have followed to overcome them.

The spectrum of the open XXZ spin 1/2 quantum chain with parallel z-oriented magnetic fields on the boundaries has been characterized in [3], in the framework of the algebraic Bethe ansatz. While its dynamics has been studied by the exact computation of correlation functions first in $[14,15]$ and then in [16], there generalizing in the ABA framework the method established in $[114,115]$ for the periodic chains. These open quantum spin chains with z-oriented boundary magnetic fields correspond in the Sklyanin's construction to the diagonal scalar solution of the reflection equation. However, the most general scalar solution of the 6-vertex/8-vertex reflection equation is non-diagonal $[11,13]$ producing unparalleled and not z-oriented boundaries magnetic fields. Under this general setting the analysis of the spectrum and dynamics has shown to be much more involved. The ABA method cannot be directly applied to these open chains with general boundary. In fact, it was first understood in [37], for the XYZ spin 1/2 open chain, that the use of the Baxter's gauge transformations [116] allows to generalize the ABA method limitedly to non-diagonal boundary matrices which satisfy one special constraint. After that, the same approach, based now on the trigonometric version of the Baxter's gauge transformations, was used in $[38,39]$ to describe the XXZ spectrum by ABA under similar constraints. Let us comment that for the XXZ spectrum the same constraint was derived independently by a pure functional method based on the use of the fusion of transfer matrices and truncations identities for the roots of unit case in [22]- [24]. This has given access to the study of the spectrum leaving however the study of the dynamics for these open models still unsolved.

Results on the spectrum for the most general unconstrained boundary conditions have been achieved only more recently and they have required the introduction of methods different from the ABA. Pure eigenvalue analysis has been implemented in [25] by a functional method leading to nested Bethe ansatz type equations similar to those previously introduced in [26]. Moreover, an ansatz for polynomial T-Q functional equations with an inhomogeneous term has been recently argued in [27]. Eigenstate construction has been first considered under these general boundary in [21] by the q-Onsager algebra formalism. A different approach, based on the generalization of the Sklyanin's separation of variables (SoV) method to the reflection algebra framework, has then lead to the complete eigenvalues and eigenstates characterization $[29,30,33-36]$, proving its equivalence to an inhomogeneous TQ functional equation [36], 
and also giving access to first computations of matrix elements of local operators [33] in the eigenstates basis.

The aim of the paper is to generalize this type of results for the 6-vertex cyclic representations. Here we solve this problem in the case of one triangular and one general boundary matrix, so that our current results define also the setup for the solution of the most general boundary case as well as the paper [33] has introduced the tools to solve the case with the most general boundary in [35].

The paper is organized as it follows. In section 2, we recall the cyclic representations of the 6-vertex Yang-Baxter algebra associated to the Bazhanov-Stroganov Lax-operator. In section 3 , we define the associated representations of the cyclic 6 -vertex reflection algebra. In section 4 , we prove the diagonalizability of the generator $\mathscr{B}_{-}(\lambda)$ of the reflection algebra generated by $\mathscr{U}_{-}(\lambda)$ for the most general $K_{-}(\lambda)$ boundary matrix while we impose one constraint on the parameters of the Bazhanov-Stroganov Lax-operator for any quantum site to make easier the explicit construction of the $\mathscr{B}_{-}$-eigenstates basis. Moreover, we compute the scalar product for the so-called separate states in the $\mathscr{B}_{-}$-eigenstates basis. In section 5 , we show that the $\mathscr{B}_{-}$-eigenstates basis is the SoV-basis for the transfer matrix spectral problem associated to the most general $K_{-}$-boundary matrix and upper triangular $K_{+}$-boundary matrix and we solve in this SoV basis this spectral problem. In section 6, we show that the SoV characterization of the transfer matrix spectrum is equivalent to inhomogeneous Baxter's like TQ-functional equation with polynomial Q-functions. We present four appendices, in the first one we extend the proof of section 4 for the diagonalizability of the operator $\mathscr{B}_{-}(\lambda)$ to the case of general values of the boundary and bulk parameters. The remaining three appendices deal with the reduction of our representations to those associated to the chiral-Potts, the sine-Gordon and the XXZ spin s-chains at the $2 s+1$ roots of unit.

\section{Cyclic representations of the 6-vertex Yang-Baxter algebra}

In this section we recall the basics of the cyclic representations of the 6-vertex Yang-Baxter algebra associated to the Bazhanov-Stroganov Lax-operator. We consider the representations defined by the tensor product of $\mathrm{N}$ local representations of the 6-vertex Yang-Baxter algebra on the local Hilbert spaces $\mathscr{R}_{n}$. Each local representation is defined as the representation of a local Weyl algebra

$$
u_{n} v_{m}=q^{\delta_{n, m}} v_{m} u_{n} \quad \forall n, m \in\{1, \ldots, \mathrm{N}\},
$$

associated to a root of unit $q$, where $u_{n}$ and $v_{n}$ are the Weyl algebra generators on the Hilbert spaces $\mathscr{R}_{n}$. Here, we assume that $u_{n}$ and $v_{n}$ are unitary operators and that it holds:

$$
u_{n}^{p}=v_{n}^{p}=1 \text { for } q=e^{-i \pi \beta^{2}},
$$

with $\beta^{2}=p^{\prime} / p, p^{\prime}$ even and $p=2 l+1$ odd. This type of representation can be defined on a $p$-dimensional linear space $\mathscr{R}_{n}$, imposing that the $v_{n}$ spectrum coincides with the $p$-roots of the unit:

$$
v_{n}|k, n\rangle=q^{k}|k, n\rangle \quad \forall(n, k) \in\{1, \ldots, \mathrm{N}\} \times\{-l, \ldots, l\} .
$$

On $\mathscr{R}_{n}$ is defined a $p$-dimensional representation of the Weyl algebra by setting:

$$
u_{n}|k, n\rangle=|k+1, n\rangle \quad \forall k \in\{-l, \ldots, l\},
$$

with the cyclicity condition:

$$
|k+p, n\rangle=|k, n\rangle .
$$


$\mathscr{R}_{n}$ is also called the right local quantum space at the site $n$ of the chain. Let $\mathscr{L}_{n}$ be the dual space of $\mathscr{R}_{n}$ then we can define the following scalar products:

$$
\left\langle k, n \mid k^{\prime}, n\right\rangle=\left((\langle k, n|)^{\dagger},\left|k^{\prime}, n\right\rangle\right) \equiv \delta_{k, k^{\prime}},
$$

for any $k, k^{\prime} \in\{-l, \ldots, l\}$.

The local generators of the cyclic 6-vertex Yang-Baxter algebra can be now defined as the elements of the following Bazhanov-Stroganov Lax operator:

$$
L_{a, n}(\lambda) \equiv\left(\begin{array}{cc}
\lambda \alpha_{n} v_{n}-\beta_{n} \lambda^{-1} v_{n}^{-1} & u_{n}\left(q^{-1 / 2} a_{n} v_{n}+q^{1 / 2} b_{n} v_{n}^{-1}\right) \\
u_{n}^{-1}\left(q^{1 / 2} c_{n} v_{n}+q^{-1 / 2} d_{n} v_{n}^{-1}\right) & \gamma_{n} v_{n} / \lambda-\delta_{n} \lambda / v_{n}
\end{array}\right)_{a} \in \operatorname{End}\left(\mathbb{C}^{2} \otimes \mathscr{R}_{n}\right),
$$

where $a$ denote the so-called auxiliary space $V_{a} \simeq \mathbb{C}^{2}$. Indeed, under the condition

$$
\gamma_{n}=a_{n} c_{n} / \alpha_{n}, \quad \delta_{n}=b_{n} d_{n} / \beta_{n},
$$

$L_{a, n}(\lambda)$ is a solution of the 6-vertex Yang-Baxter equation:

$$
R_{12}(\lambda / \mu) L_{1, n}(\lambda) L_{2, n}(\mu)=L_{2, n}(\mu) L_{1, n}(\lambda) R_{12}(\lambda / \mu),
$$

with regards to the standard 6-vertex $R$-matrix:

$$
R_{a b}(\lambda)=\left(\begin{array}{cccc}
q \lambda-q^{-1} \lambda^{-1} & & & \\
& \lambda-\lambda^{-1} & q-q^{-1} & \\
& q-q^{-1} & \lambda-\lambda^{-1} & \\
& & & q \lambda-q^{-1} \lambda^{-1}
\end{array}\right)
$$

where $a$ and $b$ denote two bidimensional spaces $V_{a}, V_{b} \equiv \mathbb{C}^{2}$ and $R_{a b}(\lambda)$ is an endomorphism on their tensor product, i.e. $R_{a b}(\lambda) \in \operatorname{End}\left(\mathbb{C}^{2} \otimes \mathbb{C}^{2}\right)$. Then, the following monodromy matrix:

$$
M_{a}(\lambda)=\left(\begin{array}{cc}
A(\lambda) & B(\lambda) \\
C(\lambda) & D(\lambda)
\end{array}\right)_{a} \equiv L_{a, \mathrm{~N}}\left(\lambda q^{-1 / 2}\right) \cdots L_{a, 1}\left(\lambda q^{-1 / 2}\right) \in \operatorname{End}\left(\mathbb{C}^{2} \otimes \mathscr{H}\right)
$$

is also a solution of the Yang-Baxter equation:

$$
R_{12}(\lambda / \mu) M_{1}(\lambda) M_{2}(\mu)=M_{2}(\mu) M_{1}(\lambda) R_{12}(\lambda / \mu),
$$

and its elements define a representation of the Yang-Baxter algebra on the tensor product of the local representation spaces, i.e. $\mathscr{H}=\otimes_{n=1}^{\mathrm{N}} \mathscr{R}_{n}$. Note that one can also consider cyclic representations of the 6-vertex Yang-Baxter algebra associated to $q$, an even root of unit, these have been studied in [117].

\subsection{Bulk transfer matrix and quantum determinant}

The Yang-Baxter equation implies that the bulk transfer matrix $\tau_{2}(\lambda) \equiv \operatorname{tr}_{a} M_{a}(\lambda)$ defines a one parameter family of commuting operators. Note that we have:

$$
\left[\tau_{2}(\lambda), \Theta\right]=0, \text { where } \Theta \equiv \prod_{n=1}^{N} v_{n} .
$$

In $[113,118]$ it was related to the analysis of the chP-model [119-126] and characterized by $\mathrm{SoV}$ in [92-97]. The Yang-Baxter equation also implies that the so-called quantum determinant is a central element and it has the following factorized form:

$$
\begin{aligned}
\operatorname{det}_{\mathrm{q}} M_{a}(\lambda) & \equiv A\left(\lambda q^{1 / 2}\right) D\left(\lambda q^{-1 / 2}\right)-B\left(\lambda q^{1 / 2}\right) C\left(\lambda q^{-1 / 2}\right) \\
& =D\left(\lambda q^{1 / 2}\right) A\left(\lambda q^{-1 / 2}\right)-C\left(\lambda q^{1 / 2}\right) B\left(\lambda q^{-1 / 2}\right) \\
& =\prod_{n=1}^{N} \operatorname{det}_{\mathrm{q}} L_{a, n}(\lambda),
\end{aligned}
$$


where the local quantum determinants read:

$$
\begin{aligned}
\operatorname{det}_{\mathrm{q}} L_{a, n}(\lambda) & \equiv\left(L_{a, n}(\lambda)\right)_{11}\left(L_{a, n}\left(\lambda q^{-1}\right)\right)_{22}-\left(L_{a, n}(\lambda)\right)_{12}\left(L_{a, n}\left(\lambda q^{-1}\right)\right)_{21} \\
& =\left(L_{a, n}(\lambda)\right)_{22}\left(L_{a, n}\left(\lambda q^{-1}\right)\right)_{11}-\left(L_{a, n}(\lambda)\right)_{21}\left(L_{a, n}\left(\lambda q^{-1}\right)\right)_{12} .
\end{aligned}
$$

They admit the following explicit form:

$$
\begin{aligned}
\operatorname{det}_{\mathrm{q}} M_{a}(\lambda) & =\prod_{n=1}^{\mathrm{N}} k_{n}\left(\frac{\lambda}{\mu_{n,+}}-\frac{\mu_{n,+}}{\lambda}\right)\left(\frac{\lambda}{\mu_{n,-}}-\frac{\mu_{n,-}}{\lambda}\right) \\
& =(-q)^{\mathrm{N}} \prod_{n=1}^{\mathrm{N}} \frac{\beta_{n} a_{n} c_{n}}{\alpha_{n}}\left(\frac{1}{\lambda}+q^{-1} \frac{b_{n} \alpha_{n}}{a_{n} \beta_{n}} \lambda\right)\left(\frac{1}{\lambda}+q^{-1} \frac{d_{n} \alpha_{n}}{c_{n} \beta_{n}} \lambda\right) \\
& =a(\lambda) d(\lambda / q),
\end{aligned}
$$

where:

$$
\begin{gathered}
k_{n} \equiv\left(a_{n} b_{n} c_{n} d_{n}\right)^{1 / 2}, \quad \mu_{n, h} \equiv \begin{cases}i q^{1 / 2}\left(a_{n} \beta_{n} / \alpha_{n} b_{n}\right)^{1 / 2} & h=+, \\
i q^{1 / 2}\left(c_{n} \beta_{n} / \alpha_{n} d_{n}\right)^{1 / 2} & h=-.\end{cases} \\
a(\lambda) \equiv a_{0} \prod_{n=1}^{\mathrm{N}}\left(\frac{\beta_{n}}{\lambda}+q^{-1} \frac{b_{n} \alpha_{n}}{a_{n}} \lambda\right), d(\lambda) \equiv \frac{(-1)^{\mathrm{N}}}{a_{0}} \prod_{n=1}^{\mathrm{N}} \frac{a_{n} c_{n}}{\alpha_{n}}\left(\frac{1}{\lambda}+q \frac{d_{n} \alpha_{n}}{c_{n} \beta_{n}} \lambda\right),
\end{gathered}
$$

and $a_{0}$ is a free non zero parameter.

\section{Cyclic representations of the 6-vertex reflection algebra}

In this section we define the most general cyclic representations of the 6-vertex reflection algebra associated to the Bazhanov-Stroganov Lax-operator. This is done by following the general procedure introduced by Sklyanin [3] which us allows to associate to any solution $M_{a}(\lambda) \in \operatorname{End}\left(\mathbb{C}^{2} \otimes \mathscr{H}\right)$ of the 6-vertex Yang-Baxter equation a solution $\mathscr{U}_{a}(\lambda) \in \operatorname{End}\left(\mathbb{C}^{2} \otimes \mathscr{H}\right)$ of the 6-vertex reflection equation:

$$
R_{12}(\lambda / \mu) \mathscr{U}_{1}(\lambda) R_{12}(\lambda \mu / q) \mathscr{U}_{2}(\mu)=\mathscr{U}_{2}(\mu) R_{12}(\lambda \mu / q) \mathscr{U}_{1}(\lambda) R_{12}(\lambda / \mu) .
$$

Here, we have defined:

$$
\mathscr{U}_{a}(\lambda)=M_{a}(\lambda) K_{a}(\lambda) \hat{M}_{a}(\lambda),
$$

where $K_{a}(\lambda ; \zeta, \kappa, \tau)$ is the most general scalar (boundary matrix) solution of the 6 -vertex reflection equation $[11,12]$ :

$$
K_{a}(\lambda ; \zeta, \kappa, \tau)=\frac{1}{\zeta-\frac{1}{\zeta}}\left(\begin{array}{cc}
\frac{\lambda \zeta}{q^{1 / 2}}-\frac{q^{1 / 2}}{\lambda \zeta} & \kappa e^{\tau}\left(\frac{\lambda^{2}}{q}-\frac{q}{\lambda^{2}}\right) \\
\kappa e^{-\tau}\left(\frac{\lambda^{2}}{q}-\frac{q}{\lambda^{2}}\right) & \frac{q^{1 / 2} \zeta}{\lambda}-\frac{\lambda}{\zeta q^{1 / 2}}
\end{array}\right)_{a},
$$

and we have defined:

$$
\hat{M}_{a}(\lambda)=(-1)^{\mathrm{N}} \sigma_{a}^{y} M_{a}^{t_{a}}(1 / \lambda) \sigma_{a}^{y} .
$$

Using this correspondence, the most general cyclic representations of the 6-vertex Yang-Baxter algebra, associated to the bulk monodromy matrix (2.11), define the most general cyclic representations of the 6-vertex reflection algebra, corresponding to the boundary monodromy matrices:

$$
\begin{aligned}
& \mathscr{U}_{a,-}(\lambda)=M_{a}(\lambda) K_{a,-}(\lambda) \hat{M}_{a}(\lambda)=\left(\begin{array}{ll}
\mathscr{A}_{-}(\lambda) & \mathscr{B}_{-}(\lambda) \\
\mathscr{C}_{-}(\lambda) & \mathscr{D}_{-}(\lambda)
\end{array}\right)_{a}, \\
& \mathscr{U}_{a,+}^{t_{a}}(\lambda)=M_{a}^{t_{a}}(\lambda) K_{a,+}^{t_{a}}(\lambda) \hat{M}_{a}^{t_{a}}(\lambda)=\left(\begin{array}{ll}
\mathscr{A}_{+}(\lambda) & \mathscr{C}_{+}(\lambda) \\
\mathscr{B}_{+}(\lambda) & \mathscr{D}_{+}(\lambda)
\end{array}\right)_{a},
\end{aligned}
$$


with $\mathscr{U}_{a,-}(\lambda)$ and $\mathscr{V}_{a,+}(\lambda) \equiv \mathscr{U}_{a,+}^{t_{a}}(-\lambda)$ both solution of the reflection equation (3.1), where:

$$
K_{a, \pm}(\lambda)=K_{a}\left(\lambda q^{(1 \pm 1) / 2} ; \zeta_{ \pm}, \kappa_{ \pm}, \tau_{ \pm}\right)=\left(\begin{array}{cc}
a_{ \pm}(\lambda) & b_{ \pm}(\lambda) \\
c_{ \pm}(\lambda) & d_{ \pm}(\lambda)
\end{array}\right)_{a},
$$

and $\zeta_{ \pm}, \delta_{ \pm}, \tau_{ \pm}$are arbitrary complex parameters.

\subsection{Boundary transfer matrix and quantum determinant}

These boundary monodromy matrices define a one parameter family of commuting transfer matrices:

$$
\begin{aligned}
\mathscr{T}(\lambda) & \equiv \operatorname{tr}_{a}\left\{K_{a,+}(\lambda) M_{a}(\lambda) K_{a,-}(\lambda) \hat{M}_{a}(\lambda)\right\} \\
& =\operatorname{tr}_{a}\left\{K_{a,-}(\lambda) \mathscr{U}_{a,+}(\lambda)\right\}=\operatorname{tr}_{a}\left\{K_{a,+}(\lambda) \mathscr{U}_{a,-}(\lambda)\right\} \\
& =a_{+}(\lambda) \mathscr{A}_{-}(\lambda)+d_{+}(\lambda) \mathscr{D}_{-}(\lambda)+b_{+}(\lambda) \mathscr{C}_{-}(\lambda)+c_{+}(\lambda) \mathscr{B}_{-}(\lambda) .
\end{aligned}
$$

This statement follows by using the reflection equation as Sklyanin has proven in [3]. The characterization of the spectrum (eigenvalues and eigenstates) of this class of transfer matrices is the main subject of this paper. In particular, we will restrict our attention to the special boundary condition $b_{+}(\lambda)=0$, which can be analyzed by implementing the SoV approach once is proven the diagonalizability of the $\mathscr{B}_{-}(\lambda)$ family of commuting operators. In order to introduce this spectral analysis we start pointing out some important properties satisfied by the generators of the reflection algebra $\mathscr{A}_{-}(\lambda), \mathscr{B}_{-}(\lambda), \mathscr{C}_{-}(\lambda)$ and $\mathscr{D}_{-}(\lambda)$.

Let us start with the following re-parametrization of the boundary parameters [22]:

$$
\left(\alpha_{-}-1 / \alpha_{-}\right)\left(\beta_{-}+1 / \beta_{-}\right) \equiv \frac{\zeta_{-}-1 / \zeta_{-}}{\kappa_{-}}, \quad\left(\alpha_{-}+1 / \alpha_{-}\right)\left(\beta_{-}-1 / \beta_{-}\right) \equiv \frac{\zeta_{-}+1 / \zeta_{-}}{\kappa_{-}} .
$$

Then we define the following functions:

$$
A_{-}(\lambda) \equiv g_{-}(\lambda) a\left(\lambda q^{-1 / 2}\right) d\left(1 /\left(q^{1 / 2} \lambda\right)\right)
$$

where:

$$
g_{-}(\lambda) \equiv \frac{\left(\lambda \alpha_{-} / q^{1 / 2}-q^{1 / 2} /\left(\lambda \alpha_{-}\right)\right)\left(\lambda \beta_{-} / q^{1 / 2}+q^{1 / 2} /\left(\lambda \beta_{-}\right)\right)}{\left(\alpha_{-}-1 / \alpha_{-}\right)\left(\beta_{-}+1 / \beta_{-}\right)} .
$$

Proposition 3.1. The following boundary quantum determinant:

$$
\begin{aligned}
\operatorname{det}_{q} \mathscr{U}_{a,-}(\lambda) & \equiv\left((\lambda / q)^{2}-(q / \lambda)^{2}\right)\left[\mathscr{A}_{-}\left(\lambda q^{1 / 2}\right) \mathscr{A}_{-}\left(q^{1 / 2} / \lambda\right)+\mathscr{B}_{-}\left(\lambda q^{1 / 2}\right) \mathscr{C}_{-}\left(q^{1 / 2} / \lambda\right)\right] \\
& =\left((\lambda / q)^{2}-(q / \lambda)^{2}\right)\left[\mathscr{D}_{-}\left(\lambda q^{1 / 2}\right) \mathscr{D}_{-}\left(q^{1 / 2} / \lambda\right)+\mathscr{C}_{-}\left(\lambda q^{1 / 2}\right) \mathscr{B}_{-}\left(q^{1 / 2} / \lambda\right)\right],
\end{aligned}
$$

is a central element in the reflection algebra, i.e.

$$
\left[\operatorname{det}_{q} \mathscr{U}_{a,-}(\lambda), \mathscr{U}_{a,-}(\mu)\right]=0
$$

and its explicit expression reads:

$$
\operatorname{det}_{q} \mathscr{U}_{a,-}(\lambda)=\left(\lambda^{2} / q^{2}-q^{2} / \lambda^{2}\right) \mathrm{A}_{-}\left(\lambda q^{1 / 2}\right) \mathrm{A}_{-}\left(q^{1 / 2} / \lambda\right) .
$$

Moreover, the generators of the reflection algebra satisfy the following properties:

$$
\mathscr{D}_{-}(\lambda)=\frac{\left(\lambda^{2} / q-q / \lambda^{2}\right)}{\left(\lambda^{2}-1 / \lambda^{2}\right)} \mathscr{A}_{-}\left(\lambda^{-1}\right)+\frac{(q-1 / q)}{\left(\lambda^{2}-1 / \lambda^{2}\right)} \mathscr{A}_{-}(\lambda)
$$

and

$$
\mathscr{B}_{-}\left(\lambda^{-1}\right)=-\frac{\left(\lambda^{2} q-1 /\left(q \lambda^{2}\right)\right)}{\left(\lambda^{2} / q-q / \lambda^{2}\right)} \mathscr{B}_{-}(\lambda), \mathscr{C}_{-}\left(\lambda^{-1}\right)=-\frac{\left(\lambda^{2} q-1 /\left(q \lambda^{2}\right)\right)}{\left(\lambda^{2} / q-q / \lambda^{2}\right)} \mathscr{C}_{-}(\lambda) .
$$


We omit the proof of this proposition as it can be derived repeating the main steps of the original Sklyanin's paper, where similar statements were proven for the case of spin $1 / 2$ representations of the 6-vertex reflection algebra. Let us introduce now the following notation:

$$
\mathscr{T}(\lambda) \equiv a_{+}(\lambda) \mathscr{A}_{-}(\lambda)+d_{+}(\lambda) \mathscr{D}_{-}(\lambda)
$$

for the diagonal part of the transfer matrix, i.e. the one associated to the diagonal elements of the matrix $\mathscr{U}_{a,-}(\lambda)$, and the coefficients:

$$
\begin{aligned}
& \mathrm{a}_{+}(\lambda) \equiv \frac{\left(\lambda^{2} q-1 /\left(q \lambda^{2}\right)\right)\left(\lambda \zeta_{+} / q^{1 / 2}-q^{1 / 2} /\left(\lambda \zeta_{+}\right)\right)}{\left(\lambda^{2}-1 / \lambda^{2}\right)\left(\zeta_{+}-1 / \zeta_{+}\right)}, \\
& \mathrm{d}_{+}(\lambda) \equiv \frac{\left(\lambda^{2} q-1 /\left(q \lambda^{2}\right)\right)\left(\zeta_{+} q^{1 / 2} / \lambda-\lambda /\left(q^{1 / 2} \zeta_{+}\right)\right)}{\left(\lambda^{2}-1 / \lambda^{2}\right)\left(\zeta_{+}-1 / \zeta_{+}\right)} .
\end{aligned}
$$

then we can prove the following:

Corollary 3.1. The most general transfer matrix admits the following symmetries:

$$
\mathscr{T}(\lambda)=\mathscr{T}(1 / \lambda), \quad \mathscr{T}(-\lambda)=\mathscr{T}(\lambda),
$$

and the diagonal part $\mathscr{T}(\lambda)$ has the following explicitly symmetric forms:

$$
\begin{aligned}
\mathscr{T}(\lambda) & \equiv a_{+}(\lambda) \mathscr{A}_{-}(\lambda)+a_{+}(1 / \lambda) \mathscr{A}_{-}(1 / \lambda) \\
& =d_{+}(\lambda) \mathscr{D}_{-}(\lambda)+d_{+}(1 / \lambda) \mathscr{D}_{-}(1 / \lambda) .
\end{aligned}
$$

\section{SoV representation of cyclic 6-vertex reflection algebra}

In this section we construct the left and right basis which diagonalize the one-parameter family of commuting operators $\mathscr{B}_{-}(\lambda)$ associated to the most general $K_{-}(\lambda)$ matrix. Here we impose one constraint on the parameters of the representation at any quantum site:

$$
b_{n}^{p}+a_{n}^{p}=0, \quad \forall n \in\{1, \ldots, \mathrm{N}\} .
$$

This is done in order to make completely explicit the construction of this basis; however, the proof of the diagonalizability of $\mathscr{B}_{-}(\lambda)$ can be done without these constraints and under completly general values of the inner boundary matrix and of the bulk parameters and it will be presented in appendix.

\subsection{Pseudo-vacuum states}

We implement the above constraints by imposing:

$$
b_{n}=-q^{2 j_{n}-1} a_{n},
$$

where for any $n \in\{1, \ldots, N\}$ we have fixed $j_{n} \in\{0, \ldots, p-1\}$, then we have:

$$
\left\langle j_{n}-1, n\left|\left(L_{a, n}\right)_{12}=\underline{0}, \quad\left(L_{a, n}\right)_{12}\right| j_{n}, n\right\rangle=\underline{0},
$$

as well as:

$$
\begin{aligned}
& \left\langle j_{n}-1, n\right|\left(L_{a, n}(\lambda)\right)_{11}=\mathrm{a}_{n}\left(\lambda q^{j_{n}-1}\right)\left\langle j_{n}-1, n\right| \\
& \left\langle j_{n}-1, n\right|\left(L_{a, n}(\lambda)\right)_{22}=\mathrm{d}_{n}\left(\lambda q^{1-j_{n}}\right)\left\langle j_{n}-1, n\right|,
\end{aligned}
$$


and

$$
\left(L_{a, n}(\lambda)\right)_{11}\left|j_{n}, n\right\rangle=\left|j_{n}, n\right\rangle \mathrm{a}_{n}\left(\lambda q^{j_{n}}\right), \quad\left(L_{a, n}(\lambda)\right)_{22}\left|j_{n}, n\right\rangle=\left|j_{n}, n\right\rangle \mathrm{d}_{n}\left(\lambda q^{-j_{n}}\right),
$$

where:

$$
\mathrm{a}_{n}(\lambda)=\lambda \alpha_{n}-\beta_{n} / \lambda, \quad \mathrm{d}_{n}(\lambda)=\gamma_{n} / \lambda-\lambda \delta_{n}
$$

which is of course compatible with the local quantum determinant at site $n$ :

$$
\begin{aligned}
\left\langle j_{n}-1, n\right| \operatorname{det}_{\mathrm{q}} L_{a, n}(\lambda) & =\left\langle j_{n}-1, n\right|\left[\left(L_{a, n}(\lambda)\right)_{11}\left(L_{a, n}(\lambda / q)\right)_{22}-\left(L_{a, n}\right)_{12}\left(L_{a, n}\right)_{21}\right] \\
& =\mathrm{a}_{n}\left(\lambda q^{j_{n}-1}\right) \mathrm{d}_{n}\left(\lambda q^{-j_{n}}\right)\left\langle j_{n}-1, n\right| \\
\operatorname{det}_{\mathrm{q}} L_{a, n}(\lambda)\left|j_{n}, n\right\rangle & =\left[\left(L_{a, n}(\lambda)\right)_{22}\left(L_{a, n}(\lambda / q)\right)_{11}-\left(L_{a, n}\right)_{21}\left(L_{a, n}\right)_{12}\right]\left|j_{n}, n\right\rangle \\
& =\left|j_{n}, n\right\rangle \mathrm{a}_{n}\left(\lambda q^{j_{n}-1}\right) \mathrm{d}_{n}\left(\lambda q^{-j_{n}}\right),
\end{aligned}
$$

being:

$$
\mathrm{a}_{n}\left(\lambda q^{j_{n}-1}\right) \mathrm{d}_{n}\left(\lambda q^{-j_{n}}\right)=-q \frac{\beta_{n} a_{n} c_{n}}{\alpha_{n}}\left(\frac{1}{\lambda}-q^{2\left(j_{n}-1\right)} \frac{\alpha_{n}}{\beta_{n}} \lambda\right)\left(\frac{1}{\lambda}+q^{-1} \frac{d_{n} \alpha_{n}}{c_{n} \beta_{n}} \lambda\right) .
$$

Then we can define the following left and right "reference states":

$$
\left\langle\Omega\left|=\otimes_{n=1}^{\mathrm{N}}\left\langle j_{n}-1, n|, \quad| \bar{\Omega}\right\rangle=\otimes_{n=1}^{\mathrm{N}}\right| j_{n}, n\right\rangle .
$$

The following properties are satisfied:

$$
\begin{gathered}
\langle\Omega| A\left(\lambda q^{1 / 2}\right)=a(\lambda)\langle\Omega|, \quad\langle\Omega| D\left(\lambda q^{1 / 2}\right)=d(\lambda)\langle\Omega|, \quad\langle\Omega| B(\lambda)=0, \quad\langle\Omega| C(\lambda) \neq \underline{0} \\
A\left(\lambda q^{1 / 2}\right)|\bar{\Omega}\rangle=|\bar{\Omega}\rangle a(\lambda q), D\left(\lambda q^{1 / 2}\right)|\bar{\Omega}\rangle=|\bar{\Omega}\rangle d(\lambda / q), B(\lambda)|\bar{\Omega}\rangle=\underline{0}, C(\lambda)|\bar{\Omega}\rangle \neq \underline{0},
\end{gathered}
$$

where it is simple to verify that as it should:

$$
a(\lambda)=\prod_{n=1}^{\mathrm{N}} \mathrm{a}_{n}\left(\lambda q^{j_{n}-1}\right), \quad d(\lambda)=\prod_{n=1}^{\mathrm{N}} \mathrm{d}_{n}\left(\lambda q^{1-j_{n}}\right),
$$

once we have fixed the free parameter:

$$
a_{0}=(-q)^{\mathrm{N}} \prod_{n=1}^{\mathrm{N}} q^{-j_{n}} .
$$

Of course, the coefficients $a(\lambda)$ and $d(\lambda)$ as well as the reference states depend on the choice of the $\mathrm{N}$-tuple $\left\{j_{1}, \ldots, j_{N}\right\}$ but for simplicity we do not write it explicitly.

\subsection{Representation of the reflection algebra in $\mathscr{B}_{-}(\lambda)$-eigenstates basis}

The left and right SoV-representations of the cyclic 6-vertex reflection algebra are now defined by constructing the left and right $\mathscr{B}_{-}(\lambda)$-eigenstates basis and by determining in this new basis the representation of the other generators of the algebra. In order to present our results we need to introduce some notations. We define the following functions parametrized by the $N$-tuples $\boldsymbol{h} \equiv\left(h_{1}, \ldots, h_{\mathrm{N}}\right) \in\{0, \ldots, p-1\}^{\mathrm{N}}$ :

$$
\mathrm{B}_{h}(\lambda) \equiv \kappa_{-} e^{\tau_{-}} \frac{\left(\lambda^{2} / q-q / \lambda^{2}\right)}{\left(\zeta_{-}-1 / \zeta_{-}\right)} a_{h}(\lambda) a_{h}(1 / \lambda),
$$

with

$$
a_{h}(\lambda) \equiv(-1)^{\mathrm{N}} \prod_{n=1}^{\mathrm{N}}\left(\alpha_{n} \beta_{n}\right)^{1 / 2}\left(\frac{\lambda}{\xi_{n}^{\left(h_{n}\right)}}-\frac{\xi_{n}^{\left(h_{n}\right)}}{\lambda}\right)
$$


where:

$$
\xi_{n}^{(h)}=\mu_{n,+} q^{h+1 / 2}, \xi_{n+\mathrm{N}}^{(h)} \equiv \xi_{n}^{(h)} \forall n \in\{1, \ldots, \mathrm{N}\}, a_{0}(\lambda)=a\left(\lambda / q^{1 / 2}\right) .
$$

Moreover, next, we will need also the following notations:

$$
\begin{aligned}
\Lambda & =\left(\lambda^{2}+1 / \lambda^{2}\right), \quad X_{b}^{\left(h_{b}\right)}=\left(\zeta_{b}^{\left(h_{b}\right)}\right)^{2}+1 /\left(\zeta_{b}^{\left(h_{b}\right)}\right)^{2}, X=q+1 / q \\
\zeta_{n}^{(h)} & =\left(\xi_{n}^{(h)}\right)^{\varphi_{n}} \text { for } h \in\{0, \ldots, p-1\} \text { and } \forall n \in\{1, \ldots, 2 \mathrm{~N}\}, \\
\varphi_{a} & =1-2 \theta(a-\mathrm{N}) \text { with } \theta(x)=\{0 \text { for } x \leq 0,1 \text { for } x>0\} .
\end{aligned}
$$

Theorem 4.1 (Left $\mathscr{B}_{-}(\lambda)$ SOV-representations). If $b_{-}(\lambda) \neq 0$ and it holds:

$$
\mu_{n,+}^{p} \neq \mu_{m,+}^{p} \quad \forall n \neq m \in\{1, \ldots, \mathrm{N}\}
$$

and

$$
\mu_{n,+}^{2 p} \neq \pm 1, \mu_{n,+}^{2} \neq q^{-2 h} \alpha_{-}^{2 \epsilon}, \mu_{n,+}^{2} \neq-q^{-2 h} \beta_{-}^{2 \epsilon}, \mu_{n,+}^{2} \neq q^{-2 \epsilon-2 h} \mu_{m,-}^{2 \epsilon}
$$

for any $\epsilon= \pm 1, h \in\{1, \ldots, p-1\}$ and $n, m \in\{1, \ldots, N\}$, then the states:

$$
\left\langle h_{1}, \ldots, h_{\mathrm{N}}\right| \equiv \frac{1}{\mathrm{~N}}\langle\Omega| \prod_{n=1}^{\mathrm{N}} \prod_{k_{n}=1}^{h_{n}} \frac{\mathscr{A}_{-}\left(1 / \xi_{n}^{\left(k_{n}-1\right)}\right)}{\mathrm{A}_{-}\left(1 / \xi_{n}^{\left(k_{n}-1\right)}\right)},
$$

where $h_{n} \in\{0, \ldots, p-1\}$ and $\mathrm{N}$ is a free normalization, define a $\mathscr{B}_{-}(\lambda)$-eigenstates basis of $\mathscr{H}^{*}$ :

$$
\langle\boldsymbol{h}| \mathscr{B}_{-}(\lambda)=\mathrm{B}_{\boldsymbol{h}}(\lambda)\langle\boldsymbol{h}| .
$$

Here we have denoted $\langle\boldsymbol{h}| \equiv\left\langle h_{1}, \ldots, h_{\mathrm{N}}\right|$. The quantum determinant and the following left action on the generic state $\langle\boldsymbol{h}|$ :

$$
\begin{aligned}
\langle\boldsymbol{h}| \mathscr{A}_{-}(\lambda) & =\sum_{a=1}^{2 \mathrm{~N}} \frac{\left(\lambda^{2} / q-q / \lambda^{2}\right)\left(\lambda \zeta_{a}^{\left(h_{a}\right)}-1 / \zeta_{a}^{\left(h_{a}\right)} \lambda\right)}{\left(\left(\zeta_{a}^{\left(h_{a}\right)}\right)^{2} / q-q /\left(\zeta_{a}^{\left(h_{a}\right)}\right)^{2}\right)\left(\left(\zeta_{a}^{\left(h_{a}\right)}\right)^{2}-1 /\left(\zeta_{a}^{\left(h_{a}\right)}\right)^{2}\right)} \prod_{\substack{\mathrm{N} \\
b \neq a=1}} \frac{\Lambda-X_{b}^{\left(h_{b}\right)}}{X_{a}^{\left(h_{a}\right)}-X_{b}^{\left(h_{b}\right)}} A_{-}\left(\zeta_{a}^{\left(h_{a}\right)}\right) \\
& \times\langle\boldsymbol{h}| T_{a}^{-\varphi_{a}}+(-1)^{\mathrm{N}} \operatorname{det}_{q} M(1) \frac{\left(\lambda / q^{1 / 2}+q^{1 / 2} / \lambda\right)}{2} \prod_{b=1}^{\mathrm{N}} \frac{\Lambda-X_{b}^{\left(h_{b}\right)}}{X-X_{b}^{\left(h_{b}\right)}}\langle\boldsymbol{h}| \\
& +(-1)^{\mathrm{N}} \frac{\left(\zeta_{-}+1 / \zeta_{-}\right)}{\left(\zeta_{-}-1 / \zeta_{-}\right)} \operatorname{det}_{q} M(i) \frac{\left(\lambda / q^{1 / 2}-q^{1 / 2} / \lambda\right)}{2} \prod_{b=1}^{\mathrm{N}} \frac{\Lambda-X_{b}^{\left(h_{b}\right)}}{X+X_{b}^{\left(h_{b}\right)}}\langle\boldsymbol{h}|
\end{aligned}
$$

where:

$$
\left\langle h_{1}, \ldots, h_{a}, \ldots, h_{\mathrm{N}}\right| T_{a}^{ \pm}=\left\langle h_{1}, \ldots, h_{a} \pm 1, \ldots, h_{\mathrm{N}}\right|,
$$

completely determine the representation of the other generators of the reflection algebra in the $\mathscr{B}_{-}(\lambda)$-eigenstates basis. Indeed, the representation of $\mathscr{D}_{-}(\lambda)$ follows from the identity (3.18) while $\mathscr{C}_{-}(\lambda)$ by the quantum determinant.

Proof. Let us write explicitly the decomposition of the reflection algebra generator:

$$
\begin{array}{r}
\mathscr{B}_{-}(\lambda)=(-1)^{\mathrm{N}}\left[-a_{-}(\lambda) A(\lambda) B(1 / \lambda)+b_{-}(\lambda) A(\lambda) A(1 / \lambda)\right. \\
\left.-c_{-}(\lambda) B(\lambda) B(1 / \lambda)+d_{-}(\lambda) B(\lambda) A(1 / \lambda)\right],
\end{array}
$$

in terms of the generators of the Yang-Baxter algebra. Then, by using the identities (4.14) it follows that $\langle\Omega|$ is a $\mathscr{B}_{-}(\lambda)$-eigenstate with non-zero eigenvalue:

$$
\langle\Omega| \mathscr{B}_{-}(\lambda) \equiv \mathrm{B}_{0}(\lambda)\langle\Omega| .
$$


Now by using the reflection algebra commutation relations:

$$
\begin{aligned}
\mathscr{A}_{-}\left(\lambda_{2}\right) \mathscr{B}_{-}\left(\lambda_{1}\right)= & \frac{\left(\lambda_{1} q / \lambda_{2}-\lambda_{2} /\left(\lambda_{1} q\right)\right)\left(\lambda_{1} \lambda_{2} / q-q /\left(\lambda_{1} \lambda_{2}\right)\right)}{\left(\lambda_{1} / \lambda_{2}-\lambda_{2} / \lambda_{1}\right)\left(\lambda_{1} \lambda_{2}-1 /\left(\lambda_{1} \lambda_{2}\right)\right)} \mathscr{B}_{-}\left(\lambda_{1}\right) \mathscr{A}_{-}\left(\lambda_{2}\right) \\
& +\frac{\left(\lambda_{1}^{2} / q-q / \lambda_{1}^{2}\right)(q-1 / q)}{\left(\lambda_{2} / \lambda_{1}-\lambda_{1} / \lambda_{2}\right)\left(\lambda_{1}^{2}-1 / \lambda_{1}^{2}\right)} \mathscr{B}_{-}\left(\lambda_{2}\right) \mathscr{A}_{-}\left(\lambda_{1}\right) \\
& -\frac{q-1 / q}{\left(\lambda_{1}^{2}-1 / \lambda_{1}^{2}\right)\left(\lambda_{1} \lambda_{2}-1 /\left(\lambda_{1} \lambda_{2}\right)\right)} \mathscr{B}_{-}\left(\lambda_{2}\right) \tilde{D}_{-}\left(\lambda_{1}\right)
\end{aligned}
$$

we can follow step by step the proof given in [99] to prove the validity of (4.26). The action of $\mathscr{A}_{-}\left(\zeta_{b}^{\left(h_{b}\right)}\right)$ for $b \in\{1, \ldots, 2 \mathrm{~N}\}$ follows from the definition of the states $\langle\mathbf{h}|$, the reflection algebra commutation relations (4.31) and the quantum determinant relations. Let us show now that the conditions (4.23) and (4.24) imply that the set of $\mathrm{p}^{\mathrm{N}}$ states $\langle\boldsymbol{h}|$ is a $\mathscr{B}_{-}(\boldsymbol{\lambda})$-eigenstates basis of $\mathscr{H}^{*}$. As by condition (4.23) each such state is associated to a different eigenvalue of $\mathscr{B}_{-}(\lambda)$ the only thing that we need to prove to get their linear independence is that each such state is nonzero. We know by construction that the state $\langle\Omega|$ is nonzero so let us assume by induction that the same is true for the state $\left\langle\boldsymbol{h}^{(0)}\right|=\left\langle h_{1}^{(0)}, \ldots, h_{\mathrm{N}}^{(0)}\right|$ with $h_{j}^{(0)} \in\{0, \ldots, p-2\}$ and let us show that $\left\langle\boldsymbol{h}_{j}^{(0)}\right|=\left\langle h_{1}^{(0)}, \ldots, h_{j}^{(0)}+1, \ldots, h_{\mathrm{N}}^{(0)}\right|$ is nonzero. We have that:

$$
\left\langle\boldsymbol{h}_{j}^{(0)}\right| \mathscr{A}_{-}\left(\xi_{j}^{\left(h_{j}^{(0)}+1\right)}\right)=A_{-}\left(\xi_{j}^{\left(h_{j}^{(0)}+1\right)}\right)\left\langle\boldsymbol{h}^{(0)}\right| \neq \underline{0} \quad j \in\{1, \ldots, N\}
$$

so that $\left\langle\boldsymbol{h}_{j}^{(0)}\right|$ is nonzero. Using this we can prove that all states $\left\langle\boldsymbol{h}^{(1)}\right|=\left\langle h_{1}^{(0)}+x_{1}, \ldots, h_{N}^{(0)}+x_{N}\right|$ with $x_{j} \in\{0,1\}$ for any $j \in\{1, \ldots, \mathrm{N}\}$ are nonzero, which just prove the validity of the induction. Finally, by using the identities:

$$
\mathscr{U}_{-}\left(q^{1 / 2}\right)=(-1)^{\mathrm{N}} \operatorname{det}_{q} M(1) I_{0}, \quad \mathscr{U}_{-}\left(i q^{1 / 2}\right)=i(-1)^{\mathrm{N}+1} \frac{\zeta_{-}+1 / \zeta_{-}}{\zeta_{-}-1 / \zeta_{-}} \operatorname{det}_{q} M(i) \sigma_{0}^{z},
$$

and remarking that $\mathscr{A}_{-}(\lambda)$ has the following functional dependence with regards to $\lambda$ :

$$
\mathscr{A}_{-}(\lambda)=\sum_{a=0}^{2 \mathrm{~N}+1} \lambda^{(2 a-2 \mathrm{~N}+1)} \mathscr{A}_{-, a},
$$

where $\mathscr{A}_{-, a} \in \operatorname{End}(\mathscr{H})$ are some fixed operators, we get our interpolation formula for its action on $\langle h|$.

Similarly, defining:

$$
\kappa_{a}^{(h)}=k\left(\zeta_{a}^{(h)}\right), \text { for } h \in\{0, \ldots, p-1\}, a \in\{1, \ldots, 2 \mathrm{~N}\},
$$

and the function:

$$
k(\lambda)=\left(\lambda^{2}-1 / \lambda^{2}\right) /\left(\lambda^{2} / q^{2}-q^{2} / \lambda^{2}\right),
$$

we have similar properties for the right representations:

Theorem 4.2 (Right $\mathscr{B}_{-}(\lambda)$ SOV-representations). If $b_{-}(\lambda) \neq 0$ and (4.23)-(4.24) are satisfied, the states:

$$
\left|h_{1}, \ldots, h_{\mathrm{N}}\right\rangle \equiv \frac{1}{\mathrm{~N}} \prod_{n=1}^{\mathrm{N}} \prod_{k_{n}=h_{n}}^{p-2} \frac{\mathscr{D}_{-}\left(\xi_{n}^{\left(k_{n}+1\right)}\right)}{\kappa_{n}^{\left(k_{n}+1\right)} \mathrm{A}_{-}\left(1 / \xi_{n}^{\left(k_{n}\right)}\right)}|\bar{\Omega}\rangle,
$$

with $\mathrm{N}$ the same coefficient as in (4.25), define a $\mathscr{B}_{-}(\lambda)$-eigenstates basis of $\mathscr{H}$ :

$$
\mathscr{B}_{-}(\lambda)|\boldsymbol{h}\rangle=|\boldsymbol{h}\rangle_{\mathrm{B}_{\boldsymbol{h}}}(\lambda) \text {. }
$$


On the generic state $|\boldsymbol{h}\rangle$, the action of the remaining reflection algebra generators follows by:

$$
\begin{aligned}
\mathscr{D}_{-}(\lambda)|\boldsymbol{h}\rangle= & \sum_{a=1}^{2 N} T_{a}^{-\varphi_{a}}|\boldsymbol{h}\rangle \frac{\left(\lambda^{2} / q-q / \lambda^{2}\right)\left(\lambda \zeta_{a}^{\left(h_{a}\right)}-1 / \zeta_{a}^{\left(h_{a}\right)} \lambda\right)}{\left(\left(\zeta_{a}^{\left(h_{a}\right)}\right)^{2} / q-q /\left(\zeta_{a}^{\left(h_{a}\right)}\right)^{2}\right)\left(\left(\zeta_{a}^{\left(h_{a}\right)}\right)^{2}-1 /\left(\zeta_{a}^{\left(h_{a}\right)}\right)^{2}\right)} \prod_{\substack{b=1 \\
b \neq a}}^{N} \frac{\Lambda-X_{b}^{\left(h_{b}\right)}}{X_{a}^{\left(h_{a}\right)}-X_{b}^{\left(h_{b}\right)}} \\
& \times D_{-}\left(\zeta_{a}^{\left(h_{a}\right)}\right)+|\boldsymbol{h}\rangle(-1)^{\mathrm{N}} \operatorname{det}_{q} M(1) \frac{\left(\lambda / q^{1 / 2}+q^{1 / 2} / \lambda\right)}{2} \prod_{b=1}^{N} \frac{\Lambda-X_{b}^{\left(h_{b}\right)}}{X-X_{b}^{\left(h_{b}\right)}} \\
& +(-1)^{\mathrm{N}+1}|\boldsymbol{h}\rangle \frac{\left(\zeta_{-}+1 / \zeta_{-}\right)}{\left(\zeta_{-}-1 / \zeta_{-}\right)} \operatorname{det}_{q} M(i) \frac{\left(\lambda / q^{1 / 2}-q^{1 / 2} / \lambda\right)}{2} \prod_{b=1}^{N} \frac{\Lambda-X_{b}^{\left(h_{b}\right)}}{X+X_{b}^{\left(h_{b}\right)}}
\end{aligned}
$$

where:

$$
D_{-}(\lambda)=k(\lambda) A_{-}(q / \lambda), \quad T_{a}^{ \pm}\left|h_{1}, \ldots, h_{a}, \ldots, h_{N}\right\rangle=\left|h_{1}, \ldots, h_{a} \pm 1, \ldots, h_{N}\right\rangle .
$$

Indeed, the representation of $\mathscr{A}_{-}(\lambda)$ follows from the identity (3.18) while $\mathscr{C}_{-}(\lambda)$ is given by the quantum determinant.

Proof. The proof is given along the same steps used in the previous theorem, we just need to make the following remarks. First of all by using the identities (4.15) it follows that $|\bar{\Omega}\rangle$ is a $\mathscr{B}_{-}(\lambda)$-eigenstate with non-zero eigenvalue:

$$
\mathscr{B}_{-}(\lambda)|\bar{\Omega}\rangle \equiv|\bar{\Omega}\rangle_{\mathrm{B}_{\mathbf{p}-1}}(\lambda) .
$$

Now all we need are the following reflection algebra commutation relations:

$$
\begin{aligned}
\mathscr{B}_{-}\left(\lambda_{1}\right) \mathscr{D}_{-}\left(\lambda_{2}\right)= & \frac{\left(\lambda_{1} q / \lambda_{2}-\lambda_{2} /\left(\lambda_{1} q\right)\right)\left(\lambda_{1} \lambda_{2} / q-q /\left(\lambda_{1} \lambda_{2}\right)\right)}{\left(\lambda_{1} / \lambda_{2}-\lambda_{2} / \lambda_{1}\right)\left(\lambda_{1} \lambda_{2}-1 /\left(\lambda_{1} \lambda_{2}\right)\right)} \mathscr{D}_{-}\left(\lambda_{2}\right) \mathscr{B}_{-}\left(\lambda_{1}\right) \\
& -\frac{(q-1 / q)\left(\lambda_{1} \lambda_{2} / q-q /\left(\lambda_{1} \lambda_{2}\right)\right)}{\left(\lambda_{1} / \lambda_{2}-\lambda_{2} / \lambda_{1}\right)\left(\lambda_{1} \lambda_{2}-1 /\left(\lambda_{1} \lambda_{2}\right)\right)} \mathscr{D}_{-}\left(\lambda_{1}\right) \mathscr{B}_{-}\left(\lambda_{2}\right) \\
& -\frac{q-1 / q}{\left(\lambda_{1} \lambda_{2}-1 /\left(\lambda_{1} \lambda_{2}\right)\right)} \mathscr{A}_{-}\left(\lambda_{1}\right) \mathscr{B}_{-}\left(\lambda_{2}\right) .
\end{aligned}
$$

By using them, the definition of the states $|\boldsymbol{h}\rangle$ and the quantum determinant, we get our interpolation formula for the right action of $\mathscr{D}_{-}(\lambda)$ on $|\boldsymbol{h}\rangle$. Let us remark that in fact, the chosen gauge for the coefficients of $\mathscr{D}_{-}(\lambda)$ is consistent with the quantum determinant condition as we have:

$$
\mathrm{D}_{-}\left(\zeta_{a}^{(h)}\right)=\kappa_{a}^{(h)} \mathrm{A}_{-}\left(q / \zeta_{a}^{(h)}\right)
$$

for any $h \in\{0, \ldots, p-1\}$ and $a \in\{1, \ldots, 2 \mathrm{~N}\}$ and

$$
\frac{\operatorname{det}_{q} \mathscr{U}_{-}\left(\xi_{a}^{(h+1 / 2)}\right)}{\left(\left(\xi_{a}^{(h+3 / 2)}\right)^{2}-1 /\left(\xi_{a}^{(h+3 / 2)}\right)^{2}\right)}=D_{-}\left(\xi_{a}^{(h+1)}\right) D_{-}\left(1 / \xi_{a}^{(h)}\right)=A_{-}\left(\xi_{a}^{(h+1)}\right) A_{-}\left(1 / \xi_{a}^{(h)}\right)
$$

since,

$$
\kappa_{a}^{(h+1)} \kappa_{a+\mathrm{N}}^{(h)}=1
$$

for any $h \in\{0, \ldots, p-1\}$ and $a \in\{1, \ldots, \mathrm{N}\}$. 


\subsection{Change of basis and SoV spectral decomposition of the identity}

In this section we present the main properties of the $p^{N} \times p^{N}$ matrices $U^{(L)}$ and $U^{(R)}$ defining respectively the change of basis from the original left and right basis, formed by the $v_{n}$-eigenstates basis:

$$
\underline{\langle\boldsymbol{h}|} \equiv \otimes_{n=1}^{\mathrm{N}}\left\langle h_{n}, n\right| \quad \text { and } \quad \underline{|\boldsymbol{h}\rangle} \equiv \otimes_{n=1}^{\mathrm{N}}\left|h_{n}, n\right\rangle,
$$

to the left and right $\mathscr{B}_{-}$-eigenstates basis:

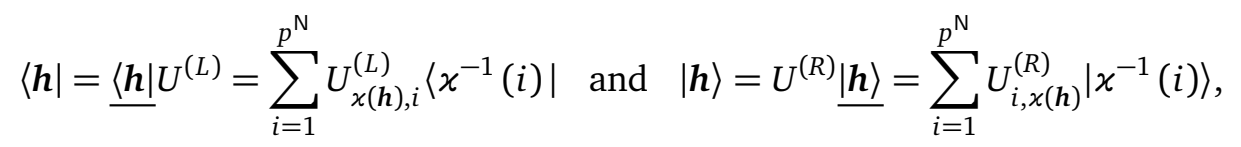

where $x$ is the isomorphism between the sets $\{0, \ldots, p-1\}^{N}$ and $\left\{1, \ldots, p^{N}\right\}$ defined by:

$$
\varkappa: \boldsymbol{h} \in\{0, \ldots, p-1\}^{\mathrm{N}} \rightarrow \chi(\boldsymbol{h}) \equiv 1+\sum_{a=1}^{\mathrm{N}} p^{(a-1)} h_{a} \in\left\{1, \ldots, p^{\mathrm{N}}\right\} .
$$

From the diagonalizability of $\mathscr{B}_{-}(\lambda)$ it follows that $U^{(L)}$ and $U^{(R)}$ are invertible matrices for which it holds:

$$
U^{(L)} \mathscr{B}_{-}(\lambda)=\Delta_{\mathscr{B}_{-}}(\lambda) U^{(L)}, \quad \mathscr{B}_{-}(\lambda) U^{(R)}=U^{(R)} \Delta_{\mathscr{B}_{-}}(\lambda)
$$

where $\Delta_{\mathscr{B}_{-}}(\lambda)$ is the $p^{\mathrm{N}} \times p^{\mathrm{N}}$ diagonal matrix defined by:

$$
\left(\Delta_{\mathscr{B}_{-}}(\lambda)\right)_{i, j} \equiv \delta_{i, j} \mathrm{~B}_{\chi^{-1}(i)}(\lambda) \forall i, j \in\left\{1, \ldots, p^{\mathrm{N}}\right\} .
$$

We can prove that it holds:

Proposition 4.1. The $p^{\mathrm{N}} \times p^{\mathrm{N}}$ matrix $M \equiv U^{(L)} U^{(R)}$ consisting of scalar products of left and right $\mathscr{B}_{-}$-eigenstates is diagonal and it is characterized by the following diagonal entries:

$$
M_{\chi(h) \chi(h)}=\langle\boldsymbol{h} \mid \boldsymbol{h}\rangle=\prod_{1 \leq b<a \leq \mathrm{N}} \frac{1}{X_{a}^{\left(h_{a}\right)}-X_{b}^{\left(h_{b}\right)}} .
$$

Proof. Note that the action of a left $\mathscr{B}_{-}$-eigenstate on a right $\mathscr{B}_{-}$-eigenstate is zero, for two different $\mathscr{B}_{-}$-eigenvalues. This implies that the matrix $M$ is diagonal; then to compute its diagonal elements we compute the matrix elements

$$
\theta_{a} \equiv\left\langle h_{1}, \ldots, h_{a}, \ldots, h_{\mathrm{N}}\left|\mathscr{A}_{-}\left(\xi_{a}^{\left(h_{a}+1\right)}\right)\right| h_{1}, \ldots, h_{a}+1, \ldots, h_{\mathrm{N}}\right\rangle \quad \text { where } \quad a \in\{1, \ldots, \mathrm{N}\} .
$$

Using the left action of the operator $\mathscr{A}_{-}\left(\xi_{a}^{\left(h_{a}+1\right)}\right)$ we get:

$$
\begin{aligned}
\theta_{a}= & \frac{(q-1 / q) \mathrm{A}_{-}\left(1 / \xi_{a}^{\left(h_{a}\right)}\right)}{\left(\left(\xi_{a}^{\left(h_{a}\right)}\right)^{2}-1 /\left(\xi_{a}^{\left(h_{a}\right)}\right)^{2}\right)} \prod_{\substack{b=1 \\
b \neq a}}^{\mathrm{N}} \frac{X_{a}^{\left(h_{a}+1\right)}-X_{b}^{\left(h_{b}\right)}}{X_{a}^{\left(h_{a}\right)}-X_{b}^{\left(h_{b}\right)}} \\
& \times\left\langle h_{1}, \ldots, h_{a}+1, \ldots, h_{\mathrm{N}} \mid h_{1}, \ldots, h_{a}+1, \ldots, h_{\mathrm{N}}\right\rangle
\end{aligned}
$$

while using the decomposition (3.18) and the fact that:

$$
\left\langle h_{1}, \ldots, h_{a}, \ldots, h_{\mathrm{N}}\left|\mathscr{D}_{-}\left(1 / \xi_{a}^{\left(h_{a}+1\right)}\right)\right| h_{1}, \ldots, h_{a}+1, \ldots, h_{\mathrm{N}}\right\rangle=0
$$

it holds:

$$
\theta_{a}=\frac{k_{a}^{\left(h_{a}+1\right)}(q-1 / q) \mathrm{A}_{-}\left(1 / \xi_{a}^{\left(h_{a}\right)}\right)}{\left(\left(\xi_{a}^{\left(h_{a}+1\right)}\right)^{2}-1 /\left(\xi_{a}^{\left(h_{a}+1\right)}\right)^{2}\right)}\left\langle h_{1}, \ldots, h_{a}, \ldots, h_{\mathrm{N}} \mid h_{1}, \ldots, h_{a}, \ldots, h_{\mathrm{N}}\right\rangle
$$


and so:

$$
\theta_{a}=\frac{(q-1 / q) \mathrm{A}_{-}\left(1 / \xi_{a}^{\left(h_{a}\right)}\right)}{\left(\left(\xi_{a}^{\left(h_{a}\right)}\right)^{2}-1 /\left(\xi_{a}^{\left(h_{a}\right)}\right)^{2}\right)}\left\langle h_{1}, \ldots, h_{a}, \ldots, h_{\mathrm{N}} \mid h_{1}, \ldots, h_{a}, \ldots, h_{\mathrm{N}}\right\rangle .
$$

These results lead to the identity:

$$
\frac{\left\langle h_{1}, \ldots, h_{a}+1, \ldots, h_{\mathrm{N}} \mid h_{1}, \ldots, h_{a}+1, \ldots, h_{\mathrm{N}}\right\rangle}{\left\langle h_{1}, \ldots, h_{a}, \ldots, h_{\mathrm{N}} \mid h_{1}, \ldots, h_{a}, \ldots, h_{\mathrm{N}}\right\rangle}=\prod_{\substack{b=1 \\ b \neq a}}^{\mathrm{N}} \frac{X_{a}^{\left(h_{a}\right)}-X_{b}^{\left(h_{b}\right)}}{X_{a}^{\left(h_{a}+1\right)}-X_{b}^{\left(h_{b}\right)}},
$$

from which one can prove:

$$
\frac{\left\langle h_{1}, \ldots, h_{\mathrm{N}} \mid h_{1}, \ldots, h_{\mathrm{N}}\right\rangle}{\langle p-1, \ldots, p-1 \mid p-1, \ldots, p-1\rangle}=\prod_{1 \leq b<a \leq \mathrm{N}} \frac{X_{a}^{(p-1)}-X_{b}^{(p-1)}}{X_{a}^{\left(h_{a}\right)}-X_{b}^{\left(h_{b}\right)}} .
$$

This proves the proposition being

$$
\langle p-1, \ldots, p-1 \mid p-1, \ldots, p-1\rangle=\prod_{1 \leq b<a \leq \mathrm{N}} \frac{1}{X_{a}^{(p-1)}-X_{b}^{(p-1)}},
$$

using the following choice of the normalization:

$$
\mathrm{N}=\left(\prod_{1 \leq b<a \leq \mathrm{N}}\left(X_{a}^{(p-1)}-X_{b}^{(p-1)}\right)\left\langle\Omega\left|\prod_{n=1}^{\mathrm{N}} \prod_{k_{n}=0}^{p-2} \frac{\mathscr{A}_{-}\left(1 / \xi_{n}^{\left(k_{n}\right)}\right)}{A_{-}\left(1 / \xi_{n}^{\left(k_{n}\right)}\right)}\right| \bar{\Omega}\right\rangle\right)^{1 / 2} .
$$

The previous theorem implies the following spectral decomposition of the identity $\mathbb{I}$ in the SoV basis:

$$
\mathbb{I} \equiv \sum_{h_{1}, \ldots, h_{N}=0}^{p-1} \prod_{1 \leq b<a \leq N}\left(X_{a}^{\left(h_{a}\right)}-X_{a}^{\left(h_{a}\right)}\right)\left|h_{1}, \ldots, h_{N}\right\rangle\left\langle h_{1}, \ldots, h_{N}\right| .
$$

\subsection{Separate states and their scalar products}

Let us introduce a class of left and right states, the so-called separate states, characterized by the following type of decompositions in the left and right SoV-basis:

$$
\begin{aligned}
& \langle\alpha|=\sum_{h_{1}, \ldots, h_{N}=0}^{p-1} \prod_{a=1}^{N} \alpha_{a}^{\left(h_{a}\right)} \prod_{1 \leq b<a \leq N}\left(X_{a}^{\left(h_{a}\right)}-X_{b}^{\left(h_{b}\right)}\right)\left\langle h_{1}, \ldots, h_{\mathrm{N}}\right|, \\
& |\beta\rangle=\sum_{h_{1}, \ldots, h_{N}=0}^{p-1} \prod_{a=1}^{N} \beta_{a}^{\left(h_{a}\right)} \prod_{1 \leq b<a \leq \mathrm{N}}\left(X_{a}^{\left(h_{a}\right)}-X_{b}^{\left(h_{b}\right)}\right)\left|h_{1}, \ldots, h_{\mathrm{N}}\right\rangle,
\end{aligned}
$$

where the coefficients $\alpha_{a}^{\left(h_{a}\right)}$ and $\beta_{a}^{\left(h_{a}\right)}$ are arbitrary complex numbers, meaning that the coefficients of these separate states have a factorized form in this basis. These separate states are interesting at least for two reasons : they admit simple determinant scalar products, as it will be shown in the next proposition, and the eigenstates of the transfer matrix are special separate states, as we will show in the next section.

Proposition 4.2. Let us take an arbitrary separate left state $\langle\alpha|$ (separate covector) and an arbitrary separate right state $|\beta\rangle$ (separate vector) then it holds:

$$
\langle\alpha \mid \beta\rangle=\operatorname{det}_{\mathrm{N}}\left\|\mathscr{M}_{a, b}^{(\alpha, \beta)}\right\| \text { with } \mathscr{M}_{a, b}^{(\alpha, \beta)} \equiv \sum_{h=0}^{p-1} \alpha_{a}^{(h)} \beta_{a}^{(h)}\left(X_{a}^{(h)}\right)^{(b-1)} .
$$


Proof. The proof follows the same method as in [97]. The formula (4.51) implies, using the representation of the states $\langle\alpha|$ and $|\beta\rangle$, the following:

$$
\langle\alpha \mid \beta\rangle=\sum_{h_{1}, \ldots, h_{N}=0}^{p-1} V\left(X_{1}^{\left(h_{1}\right)}, \ldots, X_{N}^{\left(h_{N}\right)}\right) \prod_{a=1}^{N} \alpha_{a}^{\left(h_{a}\right)} \beta_{a}^{\left(h_{a}\right)},
$$

where we have denoted by $V\left(x_{1}, \ldots, x_{N}\right) \equiv \prod_{1 \leq b<a \leq N}\left(x_{a}-x_{b}\right)$ the Vandermonde determinant. Finally, using the multilinearity of the determinant we get our result.

\section{$5 \quad \mathscr{T}$-spectrum characterization in the SoV basis}

In this section we present the complete characterization of the spectrum of the transfer matrix $\mathscr{T}(\lambda)$ associated to the cyclic representations of the 6-vertex reflection algebra. We first present some preliminary properties satisfied by all the eigenvalue functions of the transfer matrix $\mathscr{T}(\lambda)$ :

Lemma 5.1. Denote by $\Sigma_{\mathscr{T}}$ the transfer matrix spectrum, then any $\tau(\lambda) \in \Sigma_{\mathscr{T}}$ is an even function of $\lambda$ invariant under the transformation $\lambda \rightarrow 1 / \lambda$ which admits the following interpolation formula:

$$
\begin{aligned}
\tau(\lambda)= & \sum_{a=1}^{\mathrm{N}} \frac{\Lambda^{2}-X^{2}}{\left(X_{a}^{(0)}\right)^{2}-X^{2}} \prod_{\substack{b=1 \\
b \neq a}}^{\mathrm{N}} \frac{\Lambda-X_{b}^{(0)}}{X_{a}^{(0)}-X_{b}^{(0)}} \tau\left(\zeta_{a}^{(0)}\right)+(-1)^{\mathrm{N}} \frac{(\Lambda+X)}{2} \prod_{b=1}^{\mathrm{N}} \frac{\Lambda-X_{b}^{(0)}}{X-X_{b}^{(0)}} \operatorname{det}_{q} M(1) \\
& -(-1)^{\mathrm{N}} \frac{(\Lambda-X)}{2} \prod_{b=1}^{\mathrm{N}} \frac{\Lambda-X_{b}^{(0)}}{X+X_{b}^{(0)}} \frac{\left(\zeta_{+}+1 / \zeta_{+}\right)}{\left(\zeta_{+}-1 / \zeta_{+}\right)} \frac{\left(\zeta_{-}+1 / \zeta_{-}\right)}{\left(\zeta_{-}-1 / \zeta_{-}\right)} \operatorname{det}_{q} M(i) \\
& +\left(\Lambda^{2}-X^{2}\right) \tau_{\infty} \prod_{b=1}^{N}\left(\Lambda-X_{b}^{(0)}\right)
\end{aligned}
$$

where:

$$
\tau_{\infty} \equiv \frac{\kappa_{+} \kappa_{-}\left(e^{\tau_{+}-\tau_{-}} \prod_{b=1}^{N} \delta_{b} \gamma_{b}+e^{\tau_{-}-\tau_{+}} \prod_{b=1}^{N} \alpha_{b} \beta_{b}\right)}{\left(\zeta_{+}-1 / \zeta_{+}\right)\left(\zeta_{-}-1 / \zeta_{-}\right)}
$$

Proof. In the previous section, we have shown that the transformations $\lambda \rightarrow-\lambda$ and $\lambda \rightarrow 1 / \lambda$ are symmetries of the transfer matrix $\mathscr{T}(\lambda)$ so if $\tau(\lambda) \in \Sigma_{\mathscr{T}}$ then $\tau(\lambda)$ is left unchanged under these transformations. Moreover, the asymptotic of the transfer matrix can be easily derived by direct computations, it is central and it holds:

$$
\tau_{\infty}=\lim _{\log \lambda \rightarrow \pm \infty} \lambda^{\mp 2(\mathrm{~N}+2)} \mathscr{T}(\lambda) .
$$

The identities (4.33) imply after some simple computation that the transfer matrix is central in $q^{ \pm 1 / 2}$ and $i q^{ \pm 1 / 2}$ and that it holds:

$$
\mathscr{T}\left(q^{ \pm 1 / 2}\right)=(-1)^{\mathrm{N}} X \operatorname{det}_{q} M(1), \quad \mathscr{T}\left(i q^{ \pm 1 / 2}\right)=(-1)^{\mathrm{N}} X \frac{\left(\zeta_{+}+1 / \zeta_{+}\right)}{\left(\zeta_{+}-1 / \zeta_{+}\right)} \frac{\left(\zeta_{-}+1 / \zeta_{-}\right)}{\left(\zeta_{-}-1 / \zeta_{-}\right)} \operatorname{det}_{q} M(i) .
$$

The known functional form of $\mathscr{T}(\lambda)$ with regards to $\lambda$ together with this identities imply the interpolation formula in the lemma. 
The previous lemma defines the set of polynomials to which belong the transfer matrix eigenvalues; in order to completely characterize the eigenvalues we introduce now the following one-parameter family $D_{\tau}(\lambda)$ of $p \times p$ matrices:

$$
D_{\tau}(\lambda) \equiv\left(\begin{array}{cccccc}
\tau(\lambda) & -\mathrm{A}(1 / \lambda) & 0 & \ldots & 0 & -\mathrm{A}(\lambda) \\
-\mathrm{A}(q \lambda) & \tau(q \lambda) & -\mathrm{A}(1 /(q \lambda)) & 0 & \ldots & 0 \\
0 & \ddots & & & & \vdots \\
\vdots & & \ldots & & & \vdots \\
\vdots & & & \ldots & & \vdots \\
\vdots & & & & \ddots & 0 \\
0 & \ldots & 0 & -\mathrm{A}\left(q^{2 l-1} \lambda\right) & \tau\left(q^{2 l-1} \lambda\right) & -\mathrm{A}\left(1 /\left(q^{2 l-1} \lambda\right)\right) \\
-\mathrm{A}\left(1 /\left(q^{2 l} \lambda\right)\right) & 0 & \ldots & 0 & -\mathrm{A}\left(q^{2 l} \lambda\right) & \tau\left(q^{2 l} \lambda\right)
\end{array}\right),
$$

where for now $\tau(\lambda)$ is a generic function and we have defined:

$$
\mathrm{A}(\lambda)=\mathrm{a}_{+}(\lambda) A_{-}(\lambda) .
$$

Note that the coefficient $A(\lambda)$ satisfies the quantum determinant condition:

$$
\mathrm{A}\left(\lambda q^{1 / 2}\right) \mathrm{A}\left(q^{1 / 2} / \lambda\right)=\frac{\mathrm{a}_{+}\left(\lambda q^{1 / 2}\right) \mathrm{a}_{+}\left(q^{1 / 2} / \lambda\right) \operatorname{det}_{q} \mathscr{U}_{-}(\lambda)}{(\lambda / q)^{2}-(q / \lambda)^{2}} .
$$

Lemma 5.2. Let $\tau(\lambda)$ be a function of $\lambda$ invariant under the transformation $\lambda \rightarrow 1 / \lambda$ then $\operatorname{det}_{p} D_{\tau}(\lambda)$ is a function of $\lambda^{p}$ invariant under the transformation $\lambda^{p} \rightarrow 1 / \lambda^{p}$.

Proof. Let us observe that for the invariance of the function $\tau(\lambda)$ under $\lambda \rightarrow 1 / \lambda$, we have that:

$$
D_{\tau}(1 / \lambda)=O_{C} O_{R}\left(D_{\tau}(\lambda)\right)
$$

where we have denoted by $O_{R}$ the operation on a $p \times p$ matrix which exchanges the couple of rows $p-i$ with $i+2$ for any $i \in\{0, \ldots,(p-3) / 2\}$, similarly $O_{C}$ is the operation on a $p \times p$ matrix which exchanges the couple of columns $p-i$ with $i+2$ for any $i \in\{0, \ldots,(p-3) / 2\}$. It is then trivial to see that:

$$
\operatorname{det}_{p} D_{\tau}(1 / \lambda)=\operatorname{det}_{p} D_{\tau}(\lambda)
$$

Let us now observe that:

$$
D_{\tau}(\lambda q)=C_{p \rightarrow 1} R_{p \rightarrow 1}\left(D_{\tau}(\lambda)\right)
$$

where $R_{p \rightarrow 1}$ is the operation on a $p \times p$ matrix which move the last row in the first row leaving the order of the others unchanged and similarly $C_{p \rightarrow 1}$ is the operation on a $p \times p$ matrix which move the last column in the first column leaving the order of the others unchanged. This clearly implies that:

$$
\operatorname{det}_{p} D_{\tau}(q \lambda)=\operatorname{det}_{p} D_{\tau}(\lambda)
$$

which completes the proof of the lemma.

As mentioned before, we will restrict our attention to the special boundary condition $b_{+}(\lambda)=0$, for which the SoV-basis coincides with the $\mathscr{B}_{-}$-eigenstates basis. That is we consider the spectral problem of the transfer matrix:

$$
\mathscr{T}(\lambda) \equiv \mathscr{T}(\lambda)+c_{+}(\lambda) \mathscr{B}_{-}(\lambda),
$$

under the following conditions on the boundary parameters:

$$
b_{+}(\lambda)=0 \quad \text { and } \quad b_{-}(\lambda) \neq 0 .
$$


Note that the condition $b_{+}(\lambda)=0$, keeping instead if desired a $c_{+}(\lambda) \neq 0$, can be simply realized by the following renormalization of the boundary parameters $\kappa_{+}=e^{-\gamma} \kappa_{+}^{\prime}$ and $e^{\tau_{+}}=e^{\tau_{+}^{\prime}-\gamma}$ by sending $\gamma \rightarrow+\infty$. Under this limit the asymptotic of the transfer matrix reads:

$$
\tau_{\infty}=\frac{(-1)^{\mathrm{N}} \kappa_{+}^{\prime} \kappa_{-} e^{\tau_{-}-\tau_{+}^{\prime}} \prod_{b=1}^{\mathrm{N}} \alpha_{b} \beta_{b}}{\left(\zeta_{+}-1 / \zeta_{+}\right)\left(\zeta_{-}-1 / \zeta_{-}\right)}
$$

In the following we will suppress the unnecessary prime in $\kappa_{+}$and $\tau_{+}$.

Theorem 5.1. If the conditions:

$$
\mu_{n,+}^{2} \neq q^{-2 h} \zeta_{+}^{ \pm 2}, \quad \forall h \in\{1, \ldots, p-1\}, n \in\{1, \ldots, N\}
$$

and (4.23)-(4.24) are satisfied, then $\mathscr{T}(\lambda)$ has simple spectrum and $\Sigma_{\mathscr{T}}$ coincides with the set of polynomials $\tau(\lambda)$ of the form (5.1) with (5.14) which satisfy the following discrete system of equations:

$$
\operatorname{det} D_{\tau}\left(\zeta_{a}^{(0)}\right)=0, \forall a \in\{1, \ldots, N\}
$$

I) The right $\mathscr{T}$-eigenstate corresponding to $\tau(\lambda) \in \Sigma_{\mathscr{T}}$ is defined by the following decomposition in the right SoV-basis:

$$
|\tau\rangle=\sum_{h_{1}, \ldots, h_{N}=0}^{p-1} \prod_{a=1}^{N} Q_{\tau, a}^{\left(h_{a}\right)} \prod_{1 \leq b<a \leq N}\left(X_{a}^{\left(h_{a}\right)}-X_{b}^{\left(h_{b}\right)}\right)\left|h_{1}, \ldots, h_{N}\right\rangle,
$$

where the $Q_{\tau, a}^{\left(h_{a}\right)}$ are the unique nontrivial solution up to normalization of the linear homogeneous system:

$$
D_{\tau}\left(\zeta_{a}^{(0)}\right)\left(\begin{array}{c}
Q_{\tau, a}^{(0)} \\
\vdots \\
Q_{\tau, a}^{(p-1)}
\end{array}\right)=\left(\begin{array}{c}
0 \\
\vdots \\
0
\end{array}\right)
$$

II) The left $\mathscr{T}$-eigenstate corresponding to $\tau(\lambda) \in \Sigma_{\mathscr{T}}$ is defined by the following decomposition in the left SoV-basis:

$$
\langle\tau|=\sum_{h_{1}, \ldots, h_{N}=0}^{p-1} \prod_{a=1}^{N} \hat{Q}_{\tau, a}^{\left(h_{a}\right)} \prod_{1 \leq b<a \leq N}\left(X_{a}^{\left(h_{a}\right)}-X_{b}^{\left(h_{b}\right)}\right)\left\langle h_{1}, \ldots, h_{N}\right|,
$$

where the $\hat{Q}_{\tau, a}^{\left(h_{a}\right)}$ are the unique nontrivial solution up to normalization of the linear homogeneous system:

$$
\left(\begin{array}{lll}
\hat{Q}_{\tau, a}^{(0)} & \ldots & \hat{Q}_{\tau, a}^{(p-1)}
\end{array}\right)\left(\hat{D}_{\tau}\left(\zeta_{a}^{(0)}\right)\right)^{t_{0}}=\left(\begin{array}{lll}
0 & \ldots & 0
\end{array}\right),
$$

and $\hat{D}_{\tau}(\lambda)$ is the family of $p \times p$ matrices defined substituting in $D_{\tau}(\lambda)$ the coefficient $\mathrm{A}(\lambda)$ with

$$
\mathrm{D}(\lambda)=\mathrm{d}_{+}(\lambda) \mathrm{D}_{-}(\lambda) .
$$

Finally, using ideas from [97], let us note that if $\tau(\lambda) \neq \tau^{\prime}(\lambda) \in \Sigma_{\mathscr{T}}$ :

$$
\sum_{b=1}^{\mathrm{N}} \mathscr{M}_{a, b}^{\left(\tau, \tau^{\prime}\right)} x_{b}^{\left(\tau, \tau^{\prime}\right)}=0 \quad \forall a \in\{1, \ldots, \mathrm{N}\},
$$

where the $x_{b}^{\left(\tau, \tau^{\prime}\right)}$ are defined by:

$$
\tau(\lambda)-\tau^{\prime}(\lambda) \equiv\left(\Lambda^{2}-X^{2}\right) \sum_{b=1}^{N} x_{b}^{\left(\tau, \tau^{\prime}\right)} \Lambda^{b-1},
$$

which in particular implies that the action of $\langle\tau|$ on $\left|\tau^{\prime}\right\rangle$ is zero. 
Proof. The spectrum (eigenvalues and eigenstates) of the transfer matrix $\mathscr{T}(\lambda)$ in the SoV-basis is characterized by the following discrete system of equations:

$$
\tau\left(\xi_{n}^{\left(h_{n}\right)}\right) \Psi_{\tau}(\boldsymbol{h})=\mathrm{A}\left(\xi_{n}^{\left(h_{n}\right)}\right) \Psi_{\tau}\left(\mathrm{T}_{n}^{-}(\boldsymbol{h})\right)+\mathrm{A}\left(1 / \xi_{n}^{\left(h_{n}\right)}\right) \Psi_{\tau}\left(\mathrm{T}_{n}^{+}(\boldsymbol{h})\right),
$$

for any $n \in\{1, \ldots, N\}$ and $\boldsymbol{h} \in\{0, \ldots, p-1\}^{N}$, i.e. a system of $p^{N}$ Baxter-like equations in the wave-functions:

$$
\Psi_{\tau}(h) \equiv\left\langle h_{1}, \ldots, h_{N} \mid \tau\right\rangle,
$$

of the $\mathscr{T}$-eigenstate $|\tau\rangle$ associated to $\tau(\lambda) \in \Sigma_{\mathscr{T}}$, where:

$$
\mathrm{T}_{n}^{ \pm}(\boldsymbol{h}) \equiv\left(h_{1}, \ldots, h_{n} \pm 1, \ldots, h_{\mathrm{N}}\right) .
$$

This system admits the following equivalent representation as $\mathrm{N}$ linear systems of homogeneous equations:

$$
D_{\tau}\left(\xi_{n}^{(0)}\right)\left(\begin{array}{c}
\Psi_{\tau}\left(h_{1}, \ldots, h_{n}=0, \ldots, h_{\mathrm{N}}\right) \\
\Psi_{\tau}\left(h_{1}, \ldots, h_{n}=1, \ldots, h_{\mathrm{N}}\right) \\
\vdots \\
\Psi_{\tau}\left(h_{1}, \ldots, h_{n}=p-1, \ldots, h_{\mathrm{N}}\right)
\end{array}\right)=\left(\begin{array}{c}
0 \\
0 \\
\vdots \\
0
\end{array}\right),
$$

for any $n \in\{1, \ldots, N\}$ and for any $h_{m \neq n}$ in $\{0, \ldots, p-1\}$. Then the condition $\tau(\lambda) \in \Sigma_{\mathscr{T}}$ implies the compatibility equations for these linear systems, i.e. it must hold:

$$
\operatorname{det} D_{\tau}\left(\xi_{a}^{(0)}\right)=0, \forall a \in\{1, \ldots, \mathrm{N}\}
$$

Note that for the previous lemma this condition is verified also in the points $\zeta_{a}^{(0)}=1 / \xi_{a-N}^{(0)}$ for any $a \in\{N+1, \ldots, 2 N\}$. The rank of the matrices in (5.27) is $p-1$ being

$$
\mathrm{A}\left(\xi_{n}^{(h)}\right) \neq 0, \mathrm{~A}\left(1 / \xi_{n}^{(h-1)}\right) \neq 0 \quad \forall h \in\{1, \ldots, p-1\}, n \in\{1, \ldots, \mathrm{N}\},
$$

for the conditions (4.23), (4.24) and (5.15). Then (up to an overall normalization) the solution is unique and independent from the $h_{m \neq n} \in\{0, \ldots, p-1\}$ for any $n \in\{1, \ldots, N\}$. So fixing $\tau(\lambda) \in \Sigma_{\mathscr{T}}$ there exists (up to normalization) one and only one corresponding $\mathscr{T}$-eigenstate $|\tau\rangle$ with coefficients of the factorized form given in (5.17)-(5.18); i.e. the $\mathscr{T}$-spectrum is simple.

Vice versa, if $\tau(\lambda)$ is in the set of functions (5.1) and satisfies (5.16), then the state $|\tau\rangle$ defined by (5.17)-(5.18) satisfies:

$$
\left\langle h_{1}, \ldots, h_{\mathrm{N}}\left|\mathscr{T}\left(\zeta_{n}^{\left(h_{n}\right)}\right)\right| \tau\right\rangle=\tau\left(\zeta_{n}^{\left(h_{n}\right)}\right)\left\langle h_{1}, \ldots, h_{N} \mid \tau\right\rangle \quad \forall n \in\{1, \ldots, \mathrm{N}\}
$$

for any $\mathscr{B}_{-}$-eigenstate $\left\langle h_{1}, \ldots, h_{N}\right|$ and this implies:

$$
\left\langle h_{1}, \ldots, h_{N}|\mathscr{T}(\lambda)| \tau\right\rangle=\tau(\lambda)\left\langle h_{1}, \ldots, h_{N} \mid \tau\right\rangle \quad \forall \lambda \in \mathbb{C},
$$

i.e. $\tau(\lambda) \in \Sigma_{\mathscr{T}}$ and $|\tau\rangle$ is the corresponding $\mathscr{T}$-eigenstate.

For the left $\mathscr{T}$-eigenstates the proof follows as above, we just remark in this case that the matrix elements:

$$
\left\langle\tau\left|\mathscr{T}\left(\zeta_{n}^{\left(h_{n}\right)}\right)\right| h_{1}, \ldots, h_{\mathrm{N}}\right\rangle
$$

are computed in the right $\mathscr{B}_{-}$-representation:

$$
\tau\left(\zeta_{n}^{\left(h_{n}\right)}\right) \hat{\Psi}_{\tau}(\boldsymbol{h})=\mathrm{D}\left(\zeta_{n}^{\left(h_{n}\right)}\right) \hat{\Psi}_{\tau}\left(\mathrm{T}_{n}^{-}(\boldsymbol{h})\right)+\mathrm{D}\left(1 / \zeta_{n}^{\left(h_{n}\right)}\right) \hat{\Psi}_{\tau}\left(\mathrm{T}_{n}^{+}(\boldsymbol{h})\right), \quad \forall n \in\{1, \ldots, \mathrm{N}\}
$$

where:

$$
\hat{\Psi}_{\tau}(\boldsymbol{h}) \equiv\left\langle\tau \mid h_{1}, \ldots, h_{\mathrm{N}}\right\rangle, \quad \mathrm{D}(\lambda) \equiv \mathrm{d}_{+}(\lambda) \mathrm{D}_{-}(\lambda)
$$


It is simple to observe that it holds:

$$
\operatorname{det} D_{\tau}(\lambda)=\operatorname{det} \hat{D}_{\tau}(\lambda)
$$

as a consequence of the following identities:

$$
\mathrm{D}(\lambda)=\alpha(\lambda) \mathrm{A}(q / \lambda)
$$

and:

$$
\alpha(1 / \lambda) \alpha(q \lambda)=1, \quad \prod_{a=0}^{p-1} \alpha\left(\lambda q^{a}\right)=1
$$

where:

$$
\alpha(\lambda)=\frac{s(\lambda)}{s(q / \lambda)} k(\lambda), \quad s(\lambda)=\frac{\lambda^{2} q-1 /\left(q \lambda^{2}\right)}{\lambda^{2}-1 / \lambda^{2}} .
$$

Finally, the identity (5.22) is quite general in the SoV framework and can be proven also in our case of cyclic representations of the 6-vertex reflection algebra by following the same proof first given in the case of a periodic lattice [97].

For next applications it is interesting to show that we can obtain the coefficients of a left transfer matrix eigenstates in terms of those of the right one by introducing a recursion formula that produces both coefficients in terms of the transfer matrix eigenvalues. The following lemma holds:

Lemma 5.3. Let $\tau(\lambda) \in \Sigma_{\mathscr{T}}$ then we have:

$$
\left.\frac{\hat{Q}_{\tau, a}^{(h)}}{\hat{Q}_{\tau, a}^{(h-1)}}=\frac{\mathrm{A}\left(1 / \zeta_{a}^{(h-1)}\right)}{\mathrm{D}\left(1 / \zeta_{a}^{(h-1)}\right.}\right) \frac{Q_{\tau, a}^{(h)}}{Q_{\tau, a}^{(h-1)}}
$$

being:

$$
\frac{\hat{Q}_{\tau, a}^{(h)}}{\hat{Q}_{\tau, a}^{(0)}}=\frac{t_{\tau, a}^{(h)}}{\prod_{b=0}^{h-1} \mathrm{D}\left(1 / \zeta_{a}^{(b)}\right)}, \quad \frac{Q_{\tau, a}^{(h)}}{Q_{\tau, a}^{(0)}}=\frac{t_{\tau, a}^{(h)}}{\prod_{b=0}^{h-1} \mathrm{~A}\left(1 / \zeta_{a}^{(b)}\right)},
$$

where the $t_{\tau, a}^{(h)}$ are defined by the following recursion formula:

$$
t_{\tau, a}^{(h)}=\tau\left(\zeta_{a}^{(h-1)}\right) t_{\tau, a}^{(h-1)}-\frac{\operatorname{det}_{q} K_{+}\left(\xi_{a}^{(h-3 / 2)}\right) \operatorname{det}_{q} \mathscr{U}_{-}\left(\xi_{a}^{(h-3 / 2)}\right)}{\left(\xi_{a}^{(h-1 / 2)}\right)^{2}-1 /\left(\xi_{a}^{(h-1 / 2)}\right)^{2}} t_{\tau, a}^{(h-2)} \text { for } h \in\{1, \ldots, p-1\}
$$

with the following initial conditions $t_{\tau, a}^{(-1)}=0, t_{\tau, a}^{(0)}=1$.

Proof. We just need to prove the last two identities in this lemma as the first ones are simple consequences of them. Let us prove the second identity in (5.40). For $h=1$ we have:

$$
\frac{Q_{\tau, a}^{(1)}}{Q_{\tau, a}^{(0)}}=\frac{\tau\left(\zeta_{a}^{(0)}\right)}{\mathrm{A}\left(1 / \zeta_{a}^{(0)}\right)}
$$

by the SoV Baxter's like equation and the condition:

$$
\mathrm{A}\left(\zeta_{a}^{(0)}\right)=0, \mathrm{~A}\left(1 / \zeta_{a}^{(0)}\right) \neq 0
$$

This means that the second formula in (5.40) and (5.41) are both satisfied for $h=1$ once the initial conditions $t_{\tau, a}^{(-1)}=0, t_{\tau, a}^{(0)}=1$ are imposed. So let us assume that these two identities 
are satisfied for any $h \in\{1, \ldots, x\}$ and let us prove it for $h=x+1 \leq p-1$. We have that by the SoV equations it holds:

$$
\frac{Q_{\tau, a}^{(x+1)}}{Q_{\tau, a}^{(x-1)}}=\frac{\tau\left(\zeta_{a}^{(x)}\right)}{\mathrm{A}\left(1 / \zeta_{a}^{(x)}\right)} \frac{Q_{\tau, a}^{(x)}}{Q_{\tau, a}^{(x-1)}}-\frac{\mathrm{A}\left(\zeta_{a}^{(x)}\right)}{\mathrm{A}\left(1 / \zeta_{a}^{(x)}\right)}
$$

and so:

$$
\frac{Q_{\tau, a}^{(x+1)}}{Q_{\tau, a}^{(0)}}=\frac{\tau\left(\zeta_{a}^{(x)}\right)}{\mathrm{A}\left(1 / \zeta_{a}^{(x)}\right)} \frac{Q_{\tau, a}^{(x)}}{Q_{\tau, a}^{(0)}}-\frac{\mathrm{A}\left(\zeta_{a}^{(x)}\right)}{\mathrm{A}\left(1 / \zeta_{a}^{(x)}\right)} \frac{Q_{\tau, a}^{(x-1)}}{Q_{\tau, a}^{(0)}}
$$

which by using the formula (5.40) for $h=x-1$ and $h=x$ reads:

$$
\frac{Q_{\tau, a}^{(x+1)}}{Q_{\tau, a}^{(0)}}=\frac{\tau\left(\zeta_{a}^{(x)}\right) t_{\tau, a}^{(x)}-\mathrm{A}\left(\zeta_{a}^{(x)}\right) \mathrm{A}\left(1 / \zeta_{a}^{(x-1)}\right) t_{\tau, a}^{(x-1)}}{\prod_{b=0}^{x} \mathrm{~A}\left(1 / \zeta_{a}^{(b)}\right)},
$$

which just proves the formulae (5.40) and (5.41) for $h=x+1$ once we recall that:

$$
\mathrm{A}\left(\zeta_{a}^{(x)}\right) \mathrm{A}\left(1 / \zeta_{a}^{(x-1)}\right)=\frac{\operatorname{det}_{q} K_{a,+}\left(\xi_{a}^{(x-1 / 2)}\right) \operatorname{det}_{q} \mathscr{U}_{a,-}\left(\xi_{a}^{(x-1 / 2)}\right)}{\left(\xi_{a}^{(h+1 / 2)}\right)^{2}-1 /\left(\xi_{a}^{(h+1 / 2)}\right)^{2}} .
$$

The proof of the first identity in (5.40) can be done in the same way, we have just to use now that:

$$
\mathrm{D}\left(\zeta_{a}^{(x)}\right) \mathrm{D}\left(1 / \zeta_{a}^{(x-1)}\right)=\frac{\operatorname{det}_{q} K_{a,+}\left(\xi_{a}^{(x-1 / 2)}\right) \operatorname{det}_{q} \mathscr{U}_{a,-}\left(\xi_{a}^{(x-1 / 2)}\right)}{\left(\xi_{a}^{(h+1 / 2)}\right)^{2}-1 /\left(\xi_{a}^{(h+1 / 2)}\right)^{2}} .
$$

\section{Functional equation characterizing the $\mathscr{T}$-spectrum}

In this section we assume that at any quantum site the following constraints are satisfied:

$$
\begin{array}{ll}
b_{n}^{p}+a_{n}^{p}=0, & \forall n \in\{1, \ldots, \mathrm{N}\}, \\
c_{n}^{p}+d_{n}^{p}=0, & \forall n \in\{1, \ldots, \mathrm{N}\} .
\end{array}
$$

Under these conditions we can explicitly construct the left and right basis which diagonalize the one-parameter family of commuting operators $\mathscr{B}_{-}(\lambda)$ and $\mathscr{C}_{-}(\lambda)$ associated to the most general $K_{-}(\lambda)$ matrix. In the previous section we have done this construction for the $\mathscr{B}_{-}(\lambda)$ eigenstates basis, clearly we can do a similar construction also for $\mathscr{C}_{-}(\lambda)$. In the previous sections we have explained how the spectral problem of the transfer matrix $\mathscr{T}(\lambda)$ can be characterized by SoV in the $\mathscr{B}_{-}(\lambda)$ eigenstates basis when $b_{+}(\lambda)=0$ keeping instead if desired a $c_{+}(\lambda) \neq 0$, by the same approach we can characterize the $\mathscr{T}(\lambda)$-spectrum by SoV in the $\mathscr{C}_{-}(\lambda)$ eigenstates basis when $c_{+}(\lambda)=0$ keeping instead if desired a $b_{+}(\lambda) \neq 0$.

Here, we show that the SoV characterization of the $\mathscr{T}(\lambda)$-spectrum can be reformulated by functional equations. Before this, let us just observe that the central value of the asymptotic of $\mathscr{T}(\lambda)$ is given by:

$$
\tau_{\infty}=\frac{(-1)^{\mathrm{N}} \kappa_{+} \kappa_{-} e^{\epsilon\left(\tau_{-}-\tau_{+}\right)} \prod_{b=1}^{\mathrm{N}} \alpha_{b} \beta_{b}}{\left(\zeta_{+}-1 / \zeta_{+}\right)\left(\zeta_{-}-1 / \zeta_{-}\right)},
$$

where $\epsilon=+1$ for $b_{+}(\lambda)=0$ (keeping instead a $c_{+}(\lambda) \neq 0$ ) and $\epsilon=-1$ for $c_{+}(\lambda)=0$ (keeping instead a $b_{+}(\lambda) \neq 0$ ). Let us moreover introduce the following notations:

$$
\begin{aligned}
\overline{\mathrm{A}}(\lambda) & =\lambda q^{-1 / 2} \mathrm{~A}(\lambda), \\
\overline{\mathrm{A}}_{\infty} & =\lim _{\lambda \rightarrow+\infty} \lambda^{-2(\mathrm{~N}+2)} \overline{\mathrm{A}}(\lambda)=\frac{(-1)^{\mathrm{N}} \alpha_{-} \beta_{-} \zeta_{+} \kappa_{-} \prod_{n=1}^{\mathrm{N}} b_{n} c_{n}}{q^{1+\mathrm{N}}\left(\zeta_{+}-1 / \zeta_{+}\right)\left(\zeta_{-}-1 / \zeta_{-}\right)},
\end{aligned}
$$


and:

$$
F(\lambda)=\prod_{b=1}^{2 N}\left(\frac{\lambda^{p}}{\left(\zeta_{b}^{(0)}\right)^{p}}-\frac{\left(\zeta_{b}^{(0)}\right)^{p}}{\lambda^{p}}\right)
$$

then the following results hold:

Proposition 6.1. If the conditions (4.23), (4.24) and (5.15) are satisfied and:

$$
b_{n}=-q^{2 j_{n}-1} a_{n}, \quad d_{n}=-q^{2 j_{n}-1} c_{n},
$$

then $\mathscr{T}(\lambda)$ has simple spectrum and $\tau(\lambda)$ of the form (5.1) with (5.14) is an element of $\Sigma_{\mathscr{T}}$ if and only if $\operatorname{det}_{p} \bar{D}_{\tau}(\lambda)$ is a Laurent polynomial of degree $\mathrm{N}+2$ in the variable:

$$
Z=\lambda^{2 p}+\frac{1}{\lambda^{2 p}}
$$

which satisfies the following functional equation:

$$
\operatorname{det}_{p} \bar{D}_{\tau}(\lambda)-F(\lambda) \sum_{a=0}^{1} \operatorname{det}_{p} \bar{D}_{\tau}\left(i^{a} q^{1 / 2}\right) \frac{\left(\lambda^{p}+(-1)^{a} / \lambda^{p}\right)^{2}}{4(-1)^{a} F\left(i^{a} q^{1 / 2}\right)}=\left(\tau_{\infty}^{p}-\overline{\mathrm{A}}_{\infty}^{p}\right) F(\lambda)\left(\lambda^{2 p}-\frac{1}{\lambda^{2 p}}\right)^{2} .
$$

Here $\bar{D}_{\tau}(\lambda)$ is obtained from $D_{\tau}(\lambda)$ by substituting in it $\mathrm{A}(\lambda)$ with $\overline{\mathrm{A}}(\lambda)$.

Proof. Let us observe that $\operatorname{det}_{p} \bar{D}_{\tau}(\lambda)$ is an even function of $\lambda$ as a consequence of the parity of $\tau(\lambda)$ and $\bar{A}(\lambda)$ moreover following the same steps of Lemma 5.2 we can prove that $\operatorname{det}_{p} \bar{D}_{\tau}(\lambda)$ is invariant under the transformations $\lambda \rightarrow 1 / \lambda$ and $\lambda \rightarrow q \lambda$, so that $\operatorname{det}_{p} \bar{D}_{\tau}(\lambda)$ is indeed a function of $Z=\lambda^{2 p}+1 / \lambda^{2 p}$. Let us now observe that in the points $\lambda=q^{1 / 2}$ and $\lambda=i q^{1 / 2}$, the central row of the matrix $\bar{D}_{\tau}(\lambda)$ has two elements which are divergent as proportional to $\overline{\mathrm{A}}\left( \pm q^{p / 2}\right)$ and $\overline{\mathrm{A}}\left( \pm i q^{p / 2}\right)$, respectively. In the following we prove that:

$$
\operatorname{det}_{p} \bar{D}_{\tau}\left(q^{1 / 2}\right) \text { and } \operatorname{det}_{p} \bar{D}_{\tau}\left(i q^{1 / 2}\right) \text { are finite, }
$$

if we ask that the function $\tau(\lambda)$ has the functional form (5.1). Let us first introduce the notations:

$$
\bar{x}(\lambda)=\left(\lambda^{2}-\frac{1}{\lambda^{2}}\right) \bar{A}(\lambda)
$$

and using it let us expand the determinant:

$$
\begin{aligned}
\operatorname{det}_{p} \bar{D}_{\tau}\left(\lambda q^{1 / 2}\right)= & \tau(\lambda) \operatorname{det}_{p-1} \bar{D}_{\tau,(p+1) / 2,(p+1) / 2}\left(\lambda q^{1 / 2}\right)+\frac{\overline{\mathrm{x}}(\lambda) \operatorname{det}_{p-1} \bar{D}_{\tau,(p+1) / 2,(p+1) / 2-1}\left(\lambda q^{1 / 2}\right)}{\lambda^{2}-1 / \lambda^{2}} \\
& -\frac{\overline{\mathrm{x}}(1 / \lambda) \operatorname{det}_{p-1} \bar{D}_{\tau,(p+1) / 2,(p+1) / 2+1}\left(\lambda q^{1 / 2}\right)}{\lambda^{2}-1 / \lambda^{2}},
\end{aligned}
$$

with regards to the central row. Here, we have denoted with $\bar{D}_{\tau, i, j}(\lambda)$ the $(p-1) \times(p-1)$ matrix defined by removing the row $i$ and the column $j$ to the matrix $\bar{D}_{\tau}(\lambda)$. The following identity holds:

$$
\operatorname{det}_{p-1} \bar{D}_{\tau,(p+1) / 2,(p+1) / 2+1}\left(\lambda q^{1 / 2}\right)=\operatorname{det}_{p-1} \bar{D}_{\tau,(p+1) / 2,(p+1) / 2-1}\left(q^{1 / 2} / \lambda\right),
$$

and it follows that just exchanging the row $j$ with the row $p-j$, for any $j \in\{1, . .,(p+1) / 2\}$, and then the column $j$ with the column $p-j$, for any $j \in\{1, \ldots,(p+1) / 2\}$, in the matrix $\bar{D}_{\tau,(p+1) / 2,(p+1) / 2+1}\left(\lambda q^{1 / 2}\right)$. Note that the determinants $\operatorname{det}_{p-1} \bar{D}_{\tau,(p+1) / 2,(p+1) / 2+1}\left(\lambda q^{1 / 2}\right)$ and $\operatorname{det}_{p-1} \bar{D}_{\tau,(p+1) / 2,(p+1) / 2-1}\left(\lambda q^{1 / 2}\right)$ are Laurent's rational functions both finite for $\lambda \rightarrow \pm 1$ and 
$\lambda \rightarrow \pm i$. This implies that in both the limits $\lambda \rightarrow \pm 1$ and $\lambda \rightarrow \pm i$ the function $\operatorname{det}_{p} \bar{D}_{\tau}\left(\lambda q^{1 / 2}\right)$ is finite. As $Z=2$ and $Z=-2$ are the only points for which $\operatorname{det}_{p} \bar{D}_{\tau}(\lambda)$ may have divergencies, then, from $\tau(\lambda)$ and $\bar{x}(\lambda)$ Laurent's polynomial in $\lambda$ of degree $2 N+4$, it follows that $\operatorname{det}_{p} \bar{D}_{\tau}(\lambda)$ is a polynomial of degree $\mathrm{N}+2$ in the variable $Z$. Let us now remark that by the SoV characterization of the spectrum we have that $\tau(\lambda) \in \Sigma_{\mathscr{T}}$ if and only if it has the form (5.1) and satisfies:

$$
\operatorname{det}_{p} \bar{D}_{\tau}\left(\xi_{a}^{(0)}\right)=0, \forall a \in\{1, \ldots, N\},
$$

as we are assuming that (6.7) is satisfied. Finally, it is simple to verify that the following asymptotic hold:

$$
\begin{aligned}
\operatorname{det}_{p}\left[\lim _{\lambda \rightarrow+\infty} \lambda^{-2(\mathrm{~N}+2)} \bar{D}_{\tau}(\lambda)\right] & =\operatorname{det}_{p}\left[\lim _{\lambda \rightarrow 0} \lambda^{2(\mathrm{~N}+2)} \bar{D}_{\tau}(\lambda)\right]^{t} \\
& =\operatorname{det}_{p}\left(\begin{array}{cccccc}
\tau_{\infty} & 0 & 0 & \ldots & 0 & -\overline{\mathrm{A}}_{\infty} \\
-x \overline{\mathrm{A}}_{\infty} & x \tau_{\infty} & 0 & 0 & \ldots & 0 \\
0 & -x^{2} \overline{\mathrm{A}}_{\infty} & x^{2} \tau_{\infty} & \ddots & & \vdots \\
\vdots & & \ddots & \ddots & 0 & 0 \\
0 & \ldots & 0 & -x^{2 l-1} \overline{\mathrm{A}}_{\infty} & x^{2 l-1} \tau_{\infty} & 0 \\
0 & 0 & \ldots & 0 & -x^{2 l} \overline{\mathrm{A}}_{\infty} & x^{2 l} \tau_{\infty}
\end{array}\right),
\end{aligned}
$$

where we have denoted with ${ }^{t}$ the transpose of the matrix and $x=q^{2(\mathrm{~N}+2)}$, so that it holds:

$$
\lim _{\log \lambda \rightarrow \pm \infty} \lambda^{\mp 2 p(\mathrm{~N}+2)} \operatorname{det}_{p} \bar{D}_{\tau}(\lambda)=\tau_{\infty}^{p}-\overline{\mathrm{A}}_{\infty}^{p}
$$

These results fix completely the Laurent's polynomial $\operatorname{det}_{p} \bar{D}_{\tau}(\lambda)$ to satisfy (6.9).

Let us introduce now the following function:

$$
\begin{aligned}
G(\lambda \mid x, y)= & F(\lambda)\left[\left(\tau_{\infty}-q^{-2(p-1) \mathrm{N}} \overline{\mathrm{A}}_{\infty}\right) x \prod_{a=0}^{1}\left(\frac{\lambda}{i^{a} q^{1 / 2}}-\frac{i^{a} q^{1 / 2}}{\lambda}\right)\left(i^{a} q^{1 / 2} \lambda-\frac{1}{i^{a} q^{1 / 2} \lambda}\right)\right. \\
& \left.+(i-1) \frac{y \mathrm{~A}\left(i q^{1 / 2}\right)}{4 F\left(i q^{1 / 2}\right)} \prod_{a=0}^{1}\left(\lambda q^{(1-2 a) / 2}-\frac{1}{\lambda q^{(1-2 a) / 2}}\right)\right],
\end{aligned}
$$

and the states:

$$
\begin{aligned}
& \langle\omega|=\sum_{h_{1}, \ldots, h_{N}=0}^{p-1} \prod_{a=1}^{\mathrm{N}} \prod_{k_{a}=0}^{h_{a}-1} \frac{\mathrm{A}\left(1 / \zeta_{a}^{\left(k_{a}\right)}\right)}{\mathrm{D}\left(1 / \zeta_{a}^{\left(k_{a}\right)}\right)} \prod_{1 \leq b<a \leq \mathrm{N}}\left(X_{a}^{\left(h_{a}\right)}-X_{b}^{\left(h_{b}\right)}\right)\left\langle h_{1}, \ldots, h_{\mathrm{N}}\right|, \\
& |\bar{\omega}\rangle=\sum_{h_{1}, \ldots, h_{\mathrm{N}}=0}^{p-1} \prod_{1 \leq b<a \leq \mathrm{N}}\left(X_{a}^{\left(h_{a}\right)}-X_{b}^{\left(h_{b}\right)}\right)\left|h_{1}, \ldots, h_{\mathrm{N}}\right\rangle,
\end{aligned}
$$

and a renormalization of the operator

$$
\hat{\mathscr{B}}_{-}(\lambda)=\frac{\left(\zeta_{-}-1 / \zeta_{-}\right)}{\kappa_{-} e^{\tau_{-}}\left(\lambda^{2} / q-q / \lambda^{2}\right) \prod_{n=1}^{\mathrm{N}} \alpha_{n} \beta_{n}} \mathscr{B}_{-}(\lambda),
$$

which is a degree $N$ polynomial in $\Lambda$. In the following we denote with $Q(\lambda)$ a polynomial in $\Lambda=\lambda^{2}+1 / \lambda^{2}$ of degree $N_{Q} \leq(p-1) N$ which admits the following interpolation formula:

$$
Q(\lambda)=\sum_{a=1}^{(p-1) N+1} \prod_{b=1, b \neq a}^{(p-1) N+1} \frac{\Lambda-w_{b}}{w_{a}-w_{b}} Q\left(\xi_{a}\right),
$$


where $w_{b}=\xi_{b}^{2}+1 / \xi_{b}^{2}$ for any $b \in\{1, \ldots,(p-1) \mathrm{N}+1\}$ and where we have defined:

$$
\xi_{s\left(n, h_{n}\right)} \equiv \xi_{n}^{\left(h_{n}-1\right)}, s\left(n, h_{n}\right) \equiv(n-1)(p-1)+h_{n}, \forall n \in\{1, \ldots, N\}, h_{n} \in\{1, \ldots, p-1\},
$$

while $\xi_{(p-1) \mathrm{N}+1}$ is arbitrary. Moreover, we use the notation:

$$
q_{\infty} \equiv \sum_{a=1}^{(p-1) \mathrm{N}+1} \prod_{b=1, b \neq a}^{(p-1) \mathrm{N}+1} \frac{Q\left(\xi_{a}\right)}{w_{a}-w_{b}}, \quad q_{0} \equiv Q\left(i q^{1 / 2}\right) .
$$

Here, $q_{\infty}$ is the coefficient in $\Lambda^{(p-1) N}$ of the power expansion of the polynomial $Q(\lambda)$. Once this notation are introduced, the previous characterization of the spectrum can be reformulated in terms of Baxter's type TQ-functional equations and the eigenstates admit an algebraic Bethe ansatz like reformulation, as we show in the next theorem.

Theorem 6.1. Let the conditions (4.23), (4.24), (5.15) and (6.7) be satisfied and let $\tau(\lambda)$ be an entire function for which there exists a polynomial $Q(\lambda)$ of the form (6.22) satisfying the conditions:

$$
Q\left(\xi_{a}^{(0)}\right) \neq 0, \forall a \in\{1, \ldots, N\},
$$

and the following functional equation:

$$
\tau(\lambda) Q(\lambda)=\overline{\mathrm{A}}(\lambda) Q(\lambda / q)+\overline{\mathrm{A}}(1 / \lambda) Q(\lambda q)+G\left(\lambda \mid q_{\infty}, q_{0}\right),
$$

with $(p-1) \mathrm{N}-1 \leq \mathrm{N}_{Q}$, then $\tau(\lambda) \in \Sigma_{\mathscr{T}}$ and (up to normalization) the left and right transfer matrix eigenstates associated to it admit the following Bethe ansatz like representations:

$$
\left\langle\tau\left|=\left\langle\omega\left|\prod_{b=1}^{N_{Q}} \hat{\mathscr{B}}_{-}\left(\lambda_{b}\right), \quad\right| \tau\right\rangle=\prod_{b=1}^{N_{Q}} \hat{\mathscr{B}}_{-}\left(\lambda_{b}\right)\right| \bar{\omega}\right\rangle,
$$

where the $\lambda_{b}$ (fixed up the symmetry $\lambda_{b} \rightarrow-\lambda_{b}, \lambda_{b} \rightarrow 1 / \lambda_{b}$ ) for $b \in\left\{1, \ldots, N_{Q}\right\}$ are the zeros of $Q(\lambda)$. Vice versa, if $\tau(\lambda) \in \Sigma_{\mathscr{T}}$ then there exists a polynomial $Q(\lambda)$ of the form (6.22) with $(p-1) \mathrm{N}-1 \leq \mathrm{N}_{Q}$ satisfying with $\tau(\lambda)$ the Baxter's equation (6.26).

Proof. The proof of this type of reformulation of the spectrum is by now quite standard and it has been proven for several models once they admit SoV description, see for example [36, 94-96, 101-103]. So we will try to point out just some main features of the proof. Let us start proving the first part of the statement. It is simple to remark that the r.h.s. of the equation (6.26) is a Laurent polynomial in $\lambda$, indeed we can write:

$$
\overline{\mathrm{A}}(\lambda) Q(\lambda / q)+\overline{\mathrm{A}}(1 / \lambda) Q(\lambda q)=\frac{\overline{\mathrm{X}}(\lambda) Q(q / \lambda)-\overline{\mathrm{X}}(1 / \lambda) Q(\lambda q)}{\lambda^{2}-1 / \lambda^{2}}
$$

so that the limits $\lambda \rightarrow \pm 1, \lambda \rightarrow \pm i$ are all finite. Moreover, it is simple to observe that the r.h.s. of (6.26) is invariant under the transformations $\lambda \rightarrow-\lambda$ and $\lambda \rightarrow 1 / \lambda$, so that the r.h.s. of (6.26) is in fact a polynomial of degree $\mathrm{N}_{Q}+\mathrm{N}+2 \leq p \mathrm{~N}+2$ in $\Lambda$. Then, the fact that $\tau(\lambda)$ is entire in $\lambda$ implies by the equation (6.26) that $\tau(\lambda)$ is a polynomial in $\Lambda$ of the form (5.1) with (5.14). This together with the equations:

$$
\operatorname{det}_{p} \bar{D}_{\tau}\left(\xi_{a}^{(0)}\right)=0, \forall a \in\{1, \ldots, N\},
$$

which are trivial consequences of (6.26) and of (6.25), imply by the SoV characterization that $\tau(\lambda) \in \Sigma_{\mathscr{T}}$. Let us show now that the eigenstates associated to this $\tau(\lambda) \in \Sigma_{\mathscr{T}}$ can be written 
in the form (6.27). In order to do so let us observe that the $Q(\lambda)$ which is solution of (6.26) is defined up to a constant factor so that we are free to fix it by writing:

$$
Q(\lambda)=\prod_{b=1}^{N_{Q}}\left(\Lambda-\Lambda_{b}\right)
$$

where $\Lambda_{b}=\lambda_{b}^{2}+1 / \lambda_{b}^{2}$, then we have that it holds:

$$
\begin{aligned}
\prod_{b=1}^{N_{Q}} \hat{\mathscr{B}}_{-}\left(\lambda_{b}\right)|\bar{\omega}\rangle & =\sum_{h_{1}, \ldots, h_{N}=0}^{p-1} \prod_{b=1}^{\mathrm{N}_{Q}} \prod_{a=1}^{\mathrm{N}}\left(X_{a}^{\left(h_{a}\right)}-\Lambda_{b}\right) \prod_{1 \leq b<a \leq \mathrm{N}}\left(X_{a}^{\left(h_{a}\right)}-X_{b}^{\left(h_{b}\right)}\right)\left|h_{1}, \ldots, h_{\mathrm{N}}\right\rangle \\
& =\sum_{h_{1}, \ldots, h_{\mathrm{N}}=0}^{p-1} \prod_{a=1}^{\mathrm{N}} Q\left(X_{a}^{\left(h_{a}\right)}\right) \prod_{1 \leq b<a \leq \mathrm{N}}\left(X_{a}^{\left(h_{a}\right)}-X_{b}^{\left(h_{b}\right)}\right)\left|h_{1}, \ldots, h_{\mathrm{N}}\right\rangle,
\end{aligned}
$$

where in the last line appears the SoV characterization of the right eigenstate associated to $\tau(\lambda) \in \Sigma_{\mathscr{T}}$. The same steps are used to prove (6.27) for the left eigenstate. Let us comment that the polynomiality of $Q(\lambda)$ is the central property that we have used to prove these rewriting of the SoV characterizations of the transfer matrix eigenstates. This type of rewriting was first observed in the case of the noncompact XXX chains [90]. It is quite general and it has been used already for different models in the SoV framework, see for example [101-103].

Let us now assume that $\tau(\lambda) \in \Sigma_{\mathscr{T}}$ and let us prove that it exists $Q(\lambda)$ of the form (6.22). In order to do so it is enough to show that the Baxter's equation holds in $p N+2$ different values of $\Lambda$, that we chose to be $\Lambda= \pm(q+1 / q)$ and the $\Lambda=X_{a}^{\left(h_{a}\right)}$ for any $a \in\{1, \ldots, N\}$ and $h_{a} \in\{0, \ldots, p-1\}$. This set of equations can be organized in the following form:

$$
\bar{D}_{\tau}\left(\xi_{a}^{(0)}\right)\left(\begin{array}{l}
Q\left(\xi_{a}^{(h=0)}\right) \\
Q\left(\xi_{a}^{(h=1)}\right) \\
\vdots \\
\vdots \\
Q\left(\xi_{a}^{(h=p-1)}\right)
\end{array}\right)_{p \times 1}=\left(\begin{array}{l}
0 \\
\vdots \\
\vdots \\
\vdots \\
0
\end{array}\right)_{p \times 1} \quad \forall a \in\{1, \ldots, \mathrm{N}\} .
$$

They are equivalent to the following system of equations:

$$
\begin{aligned}
Q\left(\xi_{a}^{(0)}\right) \tau\left(\xi_{a}^{(0)}\right) / \overline{\mathrm{A}}\left(1 / \xi_{a}^{(0)}\right) & =Q\left(\xi_{a}^{(1)}\right) \\
\frac{\tau\left(\xi_{a}^{(h)}\right)}{\overline{\mathrm{A}}\left(1 / \xi_{a}^{(h)}\right)} Q\left(\xi_{a}^{(h)}\right)-\frac{\overline{\mathrm{A}}\left(\xi_{a}^{(h)}\right)}{\overline{\mathrm{A}}\left(1 / \xi_{a}^{(h)}\right)} Q\left(\xi_{a}^{(h-1)}\right) & =Q\left(\xi_{a}^{(h+1)}\right), \forall h \in\{1, \ldots, p-3\}, \\
\frac{\tau\left(\xi_{a}^{(p-2)}\right)}{\overline{\mathrm{A}}\left(1 / \xi_{a}^{(p-2)}\right)} Q\left(\xi_{a}^{(p-2)}\right)-\frac{\overline{\mathrm{A}}\left(\xi_{a}^{(p-2)}\right)}{\overline{\mathrm{A}}\left(1 / \xi_{a}^{(p-2)}\right)} Q\left(\xi_{a}^{(p-3)}\right) & =\prod_{b=1}^{(p-1) \mathrm{N}} \frac{X_{a}^{(p-1)}-w_{b}}{w_{(p-1) \mathrm{N}+1}-w_{b}} Q\left(\xi_{(p-1) \mathrm{N}+1}\right) \\
& +\sum_{n=1}^{\mathrm{N} Q} \prod_{\substack{b=1 \\
b \neq n}}^{(p-1) \mathrm{N}+1} \frac{X_{a}^{(p-1)}-w_{b}}{w_{n}-w_{b}} Q\left(\xi_{n}\right),
\end{aligned}
$$

as $\tau(\lambda) \in \Sigma_{\mathscr{T}}$. So we are left with (6.33)-(6.35) a linear system of $(p-1) \mathrm{N}$ inhomogeneous equations in the $(p-1) \mathrm{N}$ unknowns $Q\left(\xi_{a}\right)$ for all $a \in\{1, \ldots,(p-1) \mathrm{N}\}$. Let us define by induction the following coefficients:

$$
\mathbb{C}_{a, h+1}=x_{a, 0, h} \mathbb{C}_{a, h}+x_{a,-, h} \mathbb{C}_{a, h-1}, \quad \forall h \in\{1, \ldots, p-2\}, \mathbb{C}_{a, 1}=\tau\left(\xi_{a}^{(0)}\right) / \overline{\mathrm{A}}\left(1 / \xi_{a}^{(0)}\right), \mathbb{C}_{a, 0}=1,
$$


where we have defined:

$$
x_{a, 0, h}=\frac{\tau\left(\xi_{a}^{(h)}\right)}{\overline{\mathrm{A}}\left(1 / \xi_{a}^{(h)}\right)}, \quad x_{a,-, h}=-\frac{\overline{\mathrm{A}}\left(\xi_{a}^{(h)}\right)}{\overline{\mathrm{A}}\left(1 / \xi_{a}^{(h)}\right)}, \quad \forall h \in\{1, \ldots, p-2\}, \forall a \in\{1, \ldots, \mathrm{N}\} .
$$

One can rewrite the previous system as it follows:

$$
Q\left(\xi_{a}^{(h)}\right)=\mathbb{C}_{a, h} Q\left(\xi_{a}^{(0)}\right), \quad \forall h \in\{1, \ldots, p-1\}, \forall a \in\{1, \ldots, \mathrm{N}\},
$$

and

$$
\mathbb{C}_{a, p-1} Q\left(\xi_{a}^{(0)}\right)=\prod_{b=1}^{(p-1) \mathrm{N}} \frac{X_{a}^{(p-1)}-w_{b}}{w_{(p-1) \mathrm{N}+1}-w_{b}} Q\left(\xi_{(p-1) \mathrm{N}+1}\right)+\sum_{n=1}^{\mathrm{N}} \mathbb{D}_{a, n} Q\left(\xi_{n}^{(0)}\right),
$$

where

$$
\mathbb{D}_{b, n}=\sum_{h=0}^{p-2} \prod_{c=1, c \neq a(n, h)}^{(p-1) \mathrm{N}+1} \frac{X_{b}^{(p-1)}-w_{c}}{w_{n}-w_{c}} \mathbb{C}_{n, h}
$$

So finally we are left with a system of $\mathrm{N}$ inhomogeneous equations in the $\mathrm{N}$ unknown $Q\left(\xi_{a}^{(0)}\right)$ for all $a \in\{1, \ldots, \mathrm{N}\}$, which always admits a nontrivial solution, i.e.

$$
\left\{Q\left(\xi_{1}^{(0)}\right), \ldots, Q\left(\xi_{N}^{(0)}\right)\right\} \neq\{0, \ldots, 0\}
$$

Finally, let us point out that the degree of the polynomial $Q(\lambda)$ is constrained by the Baxter's equation (6.26). Indeed, it is trivial to remark that if $\mathrm{N}_{Q} \leq(p-1) \mathrm{N}-2$, then the equation (6.26) admits only the trivial solution $Q(\lambda)=0$ which is not compatible with equation (6.41). Instead, the equation (6.26) may still be satisfied with a nontrivial $Q(\lambda)$ if $N_{Q}=(p-1) \mathrm{N}-1$ but only if the following condition:

$$
\lim _{\Lambda \rightarrow \infty} \Lambda^{(p-1) \mathrm{N}-1} Q(\lambda)=\frac{(i-1) \mathrm{A}\left(i q^{1 / 2}\right) q_{0}}{4\left(\tau_{\infty}-q^{2-2(p-1) \mathrm{N}^{-}} \overline{\mathrm{A}}_{\infty}\right) F\left(i q^{1 / 2}\right)}
$$

is satisfied. It is clear that this represents one supplementary condition and one can expect that up to some exceptional case, related to the values of the parameters of the representation and to some special choice of the $\tau(\lambda) \in \Sigma_{\mathscr{T}}$, it is not satisfied and so that $\mathrm{N}_{Q}=(p-1) \mathrm{N}$.

It is then simple to prove the following corollary which provides under some further constraints a complete characterization of the transfer matrix eigenvalues and eigenstates in terms of solution of ordinary Bethe equations.

Corollary 6.1. If the conditions (4.23), (4.24), (5.15) and (6.7) are satisfied and if we fix the boundary parameter $\zeta_{+}=i z_{+}$with $z_{+} \in\{-1,+1\}$ and the following global condition:

$$
\frac{\kappa_{+} e^{\epsilon\left(\tau_{-}-\tau_{+}\right)}}{i z_{+} \alpha_{-} \beta_{-}}=q^{1+\mathrm{N}} \prod_{n=1}^{\mathrm{N}} \frac{b_{n} c_{n}}{\alpha_{n} \beta_{n}},
$$

where $\epsilon=+1$ for $b_{+}(\lambda)=0, c_{+}(\lambda) \neq 0$ and $\epsilon=-1$ for $c_{+}(\lambda)=0, b_{+}(\lambda) \neq 0$, then $\mathscr{T}(\lambda)$ has simple spectrum and $\tau(\lambda) \in \Sigma_{\mathscr{T}}$ if and only if $\tau(\lambda)$ is entire and there exists a polynomial $Q(\lambda)$ of the form (6.22), with $\mathrm{N}_{Q} \leq(p-1) \mathrm{N}$, which satisfies the following homogeneous Baxter equation:

$$
\tau(\lambda) Q(\lambda)=\overline{\mathrm{A}}(\lambda) Q(\lambda / q)+\overline{\mathrm{A}}(1 / \lambda) Q(\lambda q) .
$$

The $\left(\lambda_{1}, \ldots, \lambda_{N_{Q}}\right)$ entering in the Bethe ansatz like representations of the eigenstates (6.27) are solutions of the associated ordinary Bethe equations. 
Proof. The condition $\zeta_{+}=i z_{+}$with $z_{+} \in\{-1,+1\}$ implies:

$$
\tau\left(i q^{1 / 2}\right)=\mathrm{A}\left(i q^{1 / 2}\right)=0 \text {, }
$$

so that the condition (6.43) implies that $G\left(\lambda \mid q_{\infty}, q_{1}\right)=0$, for any choice of $q_{\infty}, q_{1}$. Then, the previous theorem directly implies our corollary. Finally, let us remark that in this case we do not have any restriction on the degree of the polynomial $Q(\lambda)$ imposed by the Baxter's equation (6.26), so that we are only left with $\mathrm{N}_{Q} \leq(p-1) \mathrm{N}$.

\section{Conclusions}

In this paper we have studied the transfer matrix spectrum of the class of cyclic 6-vertex representations of the reflection algebra in the case of one completely general and one triangular boundary matrices and for bulk parameters satisfying some specific constraints. Our result is the complete characterization of the spectrum (eigenvalues and eigenstates) of this class of models both by a discrete system of Baxter's like second order difference equations and by a single inhomogeneous TQ-functional equation within a class of polynomial Q-functions.

The present paper represents a natural starting point to solve the spectral problem in the most general setting. In order to do so we need to generalize to the 6-vertex cyclic representations the Baxter's gauge transformations used in the 6-vertex spin 1/2 highest weight representations and prove then the pseudo-diagonalizability of the associated family of gauge transformed $\mathscr{B}_{-}$-operators. These points are currently under analysis.

An interesting point to which we would like to come back in future investigations is the explicit construction of the Q-operator for the cyclic representations of the 6-vertex reflection algebra. This can lead to new connections with transfer matrices of exactly solvable class of models of non 6-vertex type. Indeed, let us recall that for the special class of 6-vertex cyclic representations of the Yang-Baxter algebra, parametrized by points on the algebraic curve (A.37), the associated integrable models have some remarkable connections with the inhomogeneous p-state chiral Potts models. Indeed, the chiral Potts transfer matrices play the role of the Q-operators for the transfer matrix associated to the cyclic representations of the 6-vertex Yang-Baxter algebra [113].

\section{Acknowledgements}

J. M. M. and G. N. are supported by CNRS and ENS Lyon; B. P. is supported by ENS Lyon and ENS Cachan.

\section{A Appendix}

\section{A.1 General proof of diagonalizability of $\mathscr{B}_{-}(\lambda)$}

The generator $\mathscr{B}_{-}(\lambda)$ admits the following boundary bulk decomposition:

$$
\mathscr{B}_{-}(\lambda)=-a_{-}(\lambda) A(\lambda) B(1 / \lambda)+b_{-}(\lambda) A(\lambda) A(1 / \lambda)-c_{-}(\lambda) B(\lambda) B(1 / \lambda)+d_{-}(\lambda) B(\lambda) A(1 / \lambda),
$$

if the inner boundary matrix is non-diagonal and the bulk parameters are generals, i.e. if it holds:

$$
\mathrm{B}_{-}=(-1)^{\mathrm{N}} \kappa_{-} e^{\tau_{-}} \prod_{a=1}^{\mathrm{N}} \alpha_{n} \beta_{n} \neq 0,
$$


then it trivially follows that $\mathscr{B}_{-}(\lambda)$ admits the following functional form:

$$
\mathscr{B}_{-}(\lambda)=\mathrm{B}_{-}\left(\frac{\lambda^{2}}{q}-\frac{q}{\lambda^{2}}\right) \prod_{a=1}^{\mathrm{N}}\left(\frac{\lambda}{\mathscr{B}_{-, a}}-\frac{\mathscr{B}_{-, a}}{\lambda}\right)\left(\lambda \mathscr{B}_{-, a}-\frac{1}{\lambda \mathscr{B}_{-, a}}\right),
$$

where the $\mathscr{B}_{-, a}$ are invertible commuting operators. The above functional form implies that under the condition (A.2) the operator family is not nilpotent, in particular does not exist any state annihilated by $\mathscr{B}_{-}(\lambda)$ for any $\lambda \in \mathbb{C}$. That is does not exist a reference state and we cannot use ABA to analyze the spectral problem of the transfer matrix. Here, we show that under the condition (A.2) for general values of the bulk parameters the $\mathscr{B}_{-}(\lambda)$ is indeed diagonalizable and with simple spectrum. Let us first prove the following lemma:

Lemma A.1. There exists at least one simultaneous eigenstate:

$$
\left|\Omega_{R}\right\rangle,\left\langle\Omega_{L}\right|
$$

of the one parameter family of commuting operators $\mathscr{B}_{-}(\lambda)$ :

$$
\begin{aligned}
& \mathscr{B}_{-}(\lambda)\left|\Omega_{R}\right\rangle=\left|\Omega_{R}\right\rangle \mathrm{B}_{-}\left(\frac{\lambda^{2}}{q}-\frac{q}{\lambda^{2}}\right) \prod_{a=1}^{\mathrm{N}}\left(\frac{\lambda}{\bar{b}_{-, a}}-\frac{\bar{b}_{-, a}}{\lambda}\right)\left(\lambda \bar{b}_{-, a}-\frac{1}{\lambda \bar{b}_{-, a}}\right) \\
& \left\langle\Omega_{L}\right| \mathscr{B}_{-}(\lambda)=\mathrm{B}_{-}\left(\frac{\lambda^{2}}{q}-\frac{q}{\lambda^{2}}\right) \prod_{a=1}^{\mathrm{N}}\left(\frac{\lambda}{\hat{b}_{-, a}}-\frac{\hat{b}_{-, a}}{\lambda}\right)\left(\lambda \hat{b}_{-, a}-\frac{1}{\lambda \hat{b}_{-, a}}\right)\left\langle\Omega_{L}\right| .
\end{aligned}
$$

Proof. We can always put $\mathscr{B}_{-, 1}$ in a Jordan normal form, let us denote with $\mathrm{B}_{1}$ the right eigenspace associated to a given eigenvalue $\bar{b}_{-, 1} \neq 0$ of $\mathscr{B}_{-, 1}$, as $\mathscr{B}_{-, 1}$ is invertible. If this eigenspace is one dimensional we have found our simultaneous eigenstate of $\mathscr{B}_{-}(\lambda)$. If this is not the case then by the commutativity we have that $\mathrm{B}_{1}$ is an invariant space with regards to $\mathscr{B}_{-, n}$ for any $n \in\{1, \ldots, N\}$. So we can always put $\mathscr{B}_{-, 2}$ in a Jordan normal form in $\mathrm{B}_{1}$, let us denote with $\mathrm{B}_{1,2}$ the eigenspace associated to a given eigenvalue $\bar{b}_{-, 2} \neq 0$ of $\mathscr{B}_{-, 2}$. If this eigenspace is one dimensional we have found our simultaneous eigenstate of $\mathscr{B}_{-}(\lambda)$ otherwise we can reiterate this procedure. We can have two possibilities both at the step $n \leq N$ we find that the eigenspace $\mathrm{B}_{1, \ldots, n}$ is one-dimensional or we arrive up to the eigenspace $\mathrm{B}_{1, \ldots, N}$ in both the cases we have (at least) one simultaneous eigenstate of the $\mathscr{B}_{-, n}$ for any $n \in\{1, \ldots, N\}$ and so one eigenstate of $\mathscr{B}_{-}(\lambda)$. Similarly, we can prove the existence of $\left\langle\Omega_{L}\right|$.

From the previous Lemma and the reflection algebra equation we can prove the following proposition.

Proposition A.1. Under the condition (A.2) for almost all the values of the bulk parameters, the operator family $\mathscr{B}_{-}(\lambda)$ is diagonalizable and it has simple spectrum and its average value is central and it holds:

$$
\mathbb{B}_{-}(\lambda)=\prod_{a=1}^{p} \mathscr{B}_{-}\left(\lambda q^{a}\right)=\mathrm{B}_{-}^{p}\left(\frac{\lambda^{2 p}}{1}-\frac{1}{\lambda^{2 p}}\right) \prod_{a=1}^{\mathrm{N}}\left(\frac{\lambda^{p}}{\bar{b}_{-, a}^{p}}-\frac{\bar{b}_{-, a}^{p}}{\lambda^{p}}\right)\left(\lambda^{p} \bar{b}_{-, a}^{p}-\frac{1}{\lambda p \bar{b}_{-, a}^{p}}\right),
$$

with:

$$
\bar{b}_{-, m}^{p} \neq \bar{b}_{-, n}^{p}, \forall n \neq m \in\{1, \ldots, N\} .
$$

Proof. Let us observe that by definition the operator family $\mathscr{B}_{-}(\lambda)$ is a polynomial in the bulk parameters so the same must be true for its spectrum. This implies in particular that defined

$$
\hat{b}_{n, m}=\bar{b}_{-, n}^{p}-\bar{b}_{-, m}^{p}, \forall n \neq m \in\{1, \ldots, N\},
$$


or they are identically zero or they can be zero only over subspaces of nonzero codimension in the space of the bulk parameters. So here we have just to prove that the $\hat{b}_{n, m}$ are not identically zero to derive that (A.8) holds for almost all the values of the parameters. To do so we can just recall that from the results derived in the previous section it holds:

$$
\bar{b}_{-, m}^{p}=q^{p / 2} \mu_{m,+}^{p}, \forall m \in\{1, \ldots, N\},
$$

under the condition $B(\lambda)$ nilpotent, i.e. $a_{n}^{p}=-b_{n}^{p}$ for any $n \in\{1, \ldots, N\}$. From which the condition (A.8) holds as soon as we impose:

$$
\beta_{n}^{p} / \alpha_{n}^{p} \neq \beta_{m}^{p} / \alpha_{m}^{p}, \forall n \neq m \in\{1, \ldots, N\} .
$$

Once we have proven this statement, then we have just to use the reflection algebra to construct an eigenbasis of $\mathscr{B}_{-}(\lambda)$ and this is done just by a repeated action of the generators $\mathscr{A}_{-}(\lambda)$ computed in the zeros of $\mathscr{B}_{-}(\lambda)$ on the eigenstates $\left|\Omega_{R}\right\rangle$ and $\left\langle\Omega_{L}\right|$. That is we repeat the construction of the eigenbasis presented in Section 4.2 by substituting to the reference states defined in (4.1) with the $\mathscr{B}_{-}(\lambda)$ on the eigenstates $\left|\Omega_{R}\right\rangle$ the state $\left\langle\Omega_{L}\right|$. Note that such an action can generate a null vector only if some of the zeros of $\mathscr{B}_{-}(\lambda)$ coincides with the zeros of the quantum determinant anyhow as discussed in Section 4.2 under the condition (4.24) we are always able to chose an appropriate set of $p^{N}$ zeros of $\mathscr{B}_{-}(\lambda)$ which do not coincides with those of the quantum determinant so that we generate exactly $p^{N}$ independent states.

\section{A.2 The lattice sine-Gordon model with integrable boundaries}

In this appendix we point out that the results on the transfer matrix spectrum for general cyclic representations developed in this paper apply also to characterize the spectrum of the transfer matrix of the lattice sine-Gordon model with integrable open boundary conditions. Let us recall that the Lax operator defining the lattice sine-Gordon model has the following form:

$$
L_{a, n}^{s G}\left(\lambda \mid \mathrm{U}_{n}, \mathrm{~V}_{n}, \kappa_{n}, r_{n}, s_{n}\right) \equiv\left(\begin{array}{cc}
\mathrm{U}_{n}\left(q^{-1 / 2} \kappa_{n}^{2} r_{n} s_{n} \mathrm{~V}_{n}+\frac{q^{1 / 2} s_{n}}{\mathrm{~V}_{n} r_{n}}\right) & \frac{\kappa_{n}}{i}\left(\lambda r_{n} \mathrm{~V}_{n}-\frac{1}{\lambda r_{n} \mathrm{~V}_{n}}\right) \\
\frac{\kappa_{n}}{i}\left(\frac{\lambda}{r_{n} \mathrm{~V}_{n}}-\frac{r_{n} \mathrm{v}_{n}}{\lambda}\right) & \mathrm{U}_{n}^{-1}\left(\frac{q^{1 / 2} r_{n} \mathrm{~V}_{n}}{s_{n}}+\frac{q^{-1 / 2} \kappa_{n}^{2}}{s_{n} r_{n} \mathrm{~V}_{n}}\right)
\end{array}\right),
$$

each local representation being defined as the representation of a local Weyl algebra

$$
\mathrm{U}_{n} \mathrm{~V}_{m}=q^{\delta_{n, m}} \mathrm{~V}_{m} \mathrm{U}_{n} \quad \forall n, m \in\{1, \ldots, \mathrm{N}\},
$$

associated to a root of unit $q$, where $\mathrm{U}_{n}$ and $\mathrm{v}_{n}$ are the Weyl algebra generators on the Hilbert space $\mathscr{R}_{n}$. Let us introduce the monodromy matrices for the Yang-Baxter and reflection equations of the lattice sine-Gordon model, they read:

$$
\begin{aligned}
M_{a}^{s G}(\lambda) & =L_{a, \mathrm{~N}}\left(\lambda q^{-1 / 2} / \xi_{\mathrm{N}}\right) \cdots L_{a, 1}\left(\lambda q^{-1 / 2} / \xi_{1}\right) \in \operatorname{End}\left(\mathbb{C}^{2} \otimes \mathscr{H}\right), \\
\mathscr{U}_{a,-}^{s G}(\lambda) & =M_{a}^{s G}(\lambda) K_{a,-}(\lambda) \hat{M}_{a}^{s G}(\lambda) \in \operatorname{End}\left(\mathbb{C}^{2} \otimes \mathscr{H}\right),
\end{aligned}
$$

and also the boundary transfer matrix of the sine-Gordon model, which reads:

$$
\mathscr{T}^{s G}(\lambda) \equiv t r_{a}\left\{K_{a,+}(\lambda) \mathscr{U}_{a,-}^{s G}(\lambda)\right\} .
$$

Let us remark that we have used the upper index sG in the above two monodromy matrices and transfer matrix to point out that they are related to the sine-Gordon model while we will continue to denote with $M_{a}(\lambda), \mathscr{U}_{a,-}(\lambda)$ and $\mathscr{T}(\lambda)$ those associated to the original $\tau_{2}$-model. The following lemma establishes the connection between the monodromy and transfer matrices of the sine-Gordon model and the original $\tau_{2}$-model. 
Lemma A.2. Let us denote $\mathrm{N}=2 \mathrm{M}+\mathrm{x}$ with $\mathrm{x} \in\{0,1\}$ and let us impose the following identification of the generators of the local Weyl algebras:

$$
\mathrm{U}_{2 n+\mathrm{y}}=u_{2 n+\mathrm{y}}^{(1-2 \mathrm{x})(1-2 \mathrm{y})}, \quad \mathrm{V}_{2 n+\mathrm{y}}=v_{2 n+\mathrm{y}}^{(1-2 \mathrm{x})(1-2 \mathrm{y})},
$$

with $\mathrm{y} \in\{0,1\}$ and $2 n+\mathrm{y} \in\{1, \ldots, \mathrm{N}\}$. Then the following identity holds:

$$
M_{a}^{s G}(\lambda)=M_{a}(\lambda)\left(\sigma_{a}^{x}\right)^{\times},
$$

once we define the parameters of the $\tau_{2}$-model in terms of those of the lattice sine-Gordon model as it follows:

$$
\begin{array}{lll}
a_{2 n+\mathrm{y}}=\kappa_{2 n+\mathrm{y}}^{2}\left(r_{2 n+\mathrm{y}} s_{2 n+\mathrm{y}}\right)^{(1-2 \mathrm{x})(1-2 \mathrm{y})}, & b_{2 n+\mathrm{y}}=\left(\frac{s_{2 n+\mathrm{y}}}{r_{2 n+\mathrm{y}}}\right)^{(1-2 \mathrm{x})(1-2 \mathrm{y})}, \\
c_{2 n+\mathrm{y}}=\left(\frac{r_{2 n+\mathrm{y}}}{s_{2 n+\mathrm{y}}}\right)^{(1-2 \mathrm{x})(1-2 \mathrm{y})}, & d_{2 n+\mathrm{y}}=\frac{\kappa_{2 n+\mathrm{y}}^{2}}{\left(r_{2 n+\mathrm{y}} s_{2 n+\mathrm{y}}\right)^{(1-2 \mathrm{x})(1-2 \mathrm{y})}} \\
\alpha_{2 n+\mathrm{y}}=\frac{\kappa_{2 n+\mathrm{y}} r_{2 n+\mathrm{y}}^{(1-2 \mathrm{x})(1-2 \mathrm{y})}}{i \xi_{2 n+\mathrm{y}}}, & \beta_{2 n+\mathrm{y}}=\frac{\kappa_{2 n+\mathrm{y}} \xi_{2 n+\mathrm{y}}}{i r_{2 n+\mathrm{y}}^{(1-2 \mathrm{x})(1-2 \mathrm{y})}} .
\end{array}
$$

Moreover, under the above conditions and imposing the following identifications:

$$
\tau_{\epsilon}=\epsilon^{\times} \tau_{\epsilon}^{s G}, \kappa_{\epsilon}=\epsilon^{\times} \kappa_{\epsilon}^{s G}, \zeta_{\epsilon}=\left(\zeta_{\epsilon}^{s G}\right)^{\epsilon^{\times}} \text {for } \epsilon= \pm,
$$

of the boundary parameters of the $\tau_{2}$-model and the lattice sine-Gordon model, we get:

$$
\mathscr{U}_{a,-}(\lambda)=\mathscr{U}_{a,-}^{s G}(\lambda), \quad \mathscr{T}(\lambda)=\mathscr{T}^{s G}(\lambda) .
$$

Proof. It is simple to observe that the following identities hold:

$$
L_{a, n}^{s G}\left(\lambda / \xi_{n} \mid \mathrm{U}_{n}, \mathrm{~V}_{n}, \kappa_{n}, r_{n}, s_{n}\right)=L_{a, n}(\lambda) \sigma_{a}^{x}
$$

if:

$$
\mathrm{U}_{n}=u_{n}, \quad \mathrm{v}_{n}=v_{n},
$$

and

$$
\begin{aligned}
& a_{n}=\kappa_{n}^{2} r_{n} s_{n}, \quad b_{n}=s_{n} / r_{n}, \quad c_{n}=r_{n} / s_{n}, \\
& d_{n}=\kappa_{n}^{2} /\left(r_{n} s_{n}\right), \quad \alpha_{n}=-i \kappa_{n} r_{n} / \xi_{n}, \quad \beta_{n}=-i \kappa_{n} \xi_{n} / r_{n} .
\end{aligned}
$$

Similarly by direct computations it is easy to show that defined:

$$
\tilde{L}_{a, n}^{s G}(\lambda) \equiv \sigma_{a}^{x} L_{a, n}^{s G}\left(\lambda \mid \mathrm{U}_{n}, \mathrm{~V}_{n}, \kappa_{n}, r_{n}, s_{n}\right) \sigma_{a}^{x}
$$

it holds:

$$
\tilde{L}_{a, n}^{s G}(\lambda)=L_{a, n}^{s G}\left(\lambda \mid \mathrm{U}_{n}^{-1}, \mathrm{v}_{n}^{-1}, \kappa_{n}, r_{n}^{-1}, s_{n}^{-1}\right) .
$$

Let us now observe that for $x=0$, we can write:

$$
M_{a}^{s G}(\lambda)=\left[L_{a, 2 \mathrm{M}}\left(\frac{\lambda q^{-1 / 2}}{\xi_{2 \mathrm{M}}}\right) \sigma_{a}^{x}\right]\left[\tilde{L}_{a, 2 \mathrm{M}-1}\left(\frac{\lambda q^{-1 / 2}}{\xi_{2 \mathrm{M}-1}}\right) \sigma_{a}^{x}\right] \cdots\left[L_{a, 2}\left(\frac{\lambda q^{-1 / 2}}{\xi_{2}}\right) \sigma_{a}^{x}\right]\left[\tilde{L}_{a, 1}\left(\frac{\lambda q^{-1 / 2}}{\xi_{1}}\right) \sigma_{a}^{x}\right]
$$


while for $\mathrm{x}=1$, we can write:

$$
M_{a}^{s G}(\lambda) \sigma_{a}^{x}=\left[L_{a, 2 \mathrm{M}+1}\left(\frac{\lambda q^{-1 / 2}}{\xi_{2 \mathrm{M}+1}}\right) \sigma_{a}^{x}\right]\left[\tilde{L}_{a, 2 \mathrm{M}}\left(\frac{\lambda q^{-1 / 2}}{\xi_{2 \mathrm{M}}}\right) \sigma_{a}^{x}\right] \cdots\left[\tilde{L}_{a, 2}\left(\frac{\lambda q^{-1 / 2}}{\xi_{2}}\right) \sigma_{a}^{x}\right]\left[L_{a, 1}\left(\frac{\lambda q^{-1 / 2}}{\xi_{1}}\right) \sigma_{a}^{x}\right],
$$

then these two identities together with the identity (A.24), (A.29) and the parametrization (A.26)-(A.27) imply the identity (A.18) with the parametrization (A.19)-(A.21)and the local operator identification (A.17). Finally, let us observe that from the identity (A.18) it follows:

$$
\hat{M}_{a}^{s G}(\lambda) \equiv(-1)^{\mathrm{N}} \sigma_{a}^{y}\left(M_{a}^{s G}(1 / \lambda)\right)^{t_{0}} \sigma_{a}^{y}=\left(-\sigma_{a}^{x}\right)^{\mathrm{x}} \hat{M}_{a}(\lambda),
$$

which together with:

$$
K_{a,+}\left(\lambda \mid \tau_{\epsilon}, \kappa_{\epsilon}, \zeta_{\epsilon}\right)=\left(\sigma_{a}^{x}\right)^{\times} K_{a,+}^{s G}\left(\lambda \mid \tau_{-}^{s G}, \kappa_{-}^{s G}, \zeta_{-}^{s G}\right)\left(-\sigma_{a}^{x}\right)^{\times},
$$

holding under the parametrization (A.22), implies the identities (A.23).

\section{A.3 Reduction to inhomogeneous chiral Potts representations}

In this appendix we want to point out that a nontrivial class of representations of the inhomogeneous chiral Potts model can be described on the closed chain in the space of the parameters of the $\tau_{2}$-model considered in this paper. In order to do so let us recall that the transfer matrix $\mathrm{T}_{\lambda}^{\text {chP }}$ of the inhomogeneous chiral Potts model [113] is characterized by the following kernel:

$$
\mathrm{T}_{\lambda}^{\mathrm{chP}}\left(\mathrm{z}, \mathrm{z}^{\prime}\right) \equiv\left\langle\mathrm{z}\left|\mathrm{T}_{\lambda}^{\mathrm{chP}}\right| \mathrm{z}^{\prime}\right\rangle=\prod_{n=1}^{\mathrm{N}} W_{\mathrm{q}_{n} \mathrm{p}}\left(z_{n} / z_{n}^{\prime}\right) \bar{W}_{\mathrm{r}_{n} \mathrm{p}}\left(z_{n} / z_{n+1}^{\prime}\right),
$$

in the basis $\langle z| \equiv\left\langle z_{1}, \ldots, z_{N}\right|$ and $|z\rangle \equiv\left|z_{1}, \ldots, z_{N}\right\rangle$ formed by the left and right $u_{n}$-eigenstates:

$$
\left\langle z\left|u_{n}=z_{n}\left\langle z\left|, \quad u_{n}\right| \mathrm{z}\right\rangle=z_{n}\right| \mathrm{z}\right\rangle \text { with } z_{n} \in \mathbb{S}_{p} \equiv\left\{q^{2 r}, r=1, . ., p\right\},
$$

and where:

$$
\lambda=t_{\mathrm{p}}^{-1 / 2} \mathrm{c}_{0}, \quad \mathrm{p}, \mathrm{r}_{n}, \mathrm{q}_{n} \in \mathscr{C}_{k}, \mathrm{c}_{0} \in \mathbb{C} .
$$

The algebraic curve $\mathscr{C}_{k}$ of modulus $k$ is by definition the locus of the points in the fourdimensional complex space $p \equiv\left(a_{\mathrm{p}}, b_{\mathrm{p}}, c_{\mathrm{p}}, d_{\mathrm{p}}\right) \in \mathbb{C}^{4}$ which satisfy the equations:

$$
x_{\mathrm{p}}^{p}+y_{\mathrm{p}}^{p}=k\left(1+x_{\mathrm{p}}^{p} y_{\mathrm{p}}^{p}\right), \quad k x_{\mathrm{p}}^{p}=1-k^{\prime} s_{\mathrm{p}}^{-p}, \quad k y_{\mathrm{p}}^{p}=1-k^{\prime} s_{\mathrm{p}}^{p},
$$

where:

$$
x_{\mathrm{p}} \equiv a_{\mathrm{p}} / d_{\mathrm{p}}, \quad y_{\mathrm{p}} \equiv b_{\mathrm{p}} / c_{\mathrm{p}}, \quad s_{\mathrm{p}} \equiv d_{\mathrm{p}} / c_{\mathrm{p}}, t_{\mathrm{p}} \equiv x_{\mathrm{p}} y_{\mathrm{p}}, \quad k^{2}+\left(k^{\prime}\right)^{2}=1,
$$

and $W_{\mathrm{qp}}(z(n))$ and $\bar{W}_{\mathrm{qp}}(z(n))$ are the Boltzmann weights of the chiral Potts model. Then, $\mathrm{T}_{\lambda}^{\text {chP }}$ is a Baxter $\mathrm{Q}$-operator with regards to the bulk $\tau_{2}$-transfer matrix in $\mathscr{H}_{\mathrm{N}}$.

Let us here directly characterize the class of the inhomogeneous chiral Potts representations once we restrict the space of the parameters to that used in the section 6; in particular, we assume that it holds:

$$
b_{n}=-q^{-1} a_{n}, \quad d_{n}=-q^{-1} c_{n} .
$$

The parameters of the $\tau_{2}$-Lax operators are written in terms of the coordinate of the points $\mathrm{p}$, $\mathrm{r}_{n}, \mathrm{q}_{n}$ by using the equations (5.3) of the paper [96]. Then we have that the points $\mathrm{r}_{n}, \mathrm{q}_{n}$ are elements of $\mathscr{C}_{k}$ if and only if beyond (A.39) the parameters of the $\tau_{2}$-Lax operators satisfy the following conditions:

$$
\alpha_{n} \beta_{n}=a_{n} c_{n}
$$


and

$$
\left(\frac{c_{0} \alpha_{n}}{q^{1 / 2} a_{n}}\right)^{p}+\left(\frac{q^{1 / 2} c_{0} \alpha_{n}}{c_{n}}\right)^{p}=k\left(1+\left(\frac{c_{0}^{2} \alpha_{n}^{2}}{c_{n} a_{n}}\right)^{p}\right) .
$$

Under these constraints the class of the inhomogeneous chiral Potts representations is characterized by the following identity:

$$
\mathrm{r}_{n}=\Delta\left(\mathrm{q}_{n}\right), \quad \forall n \in\{1, \ldots, \mathrm{N}\},
$$

where the $\mathrm{q}_{n}$ are free elements of $\mathscr{C}_{k}$ and $\Delta$ is the following discrete automorphism of the curve:

$$
\Delta: \mathrm{x}=\left(a_{\mathrm{x}}, b_{\mathrm{x}}, c_{\mathrm{x}}, d_{\mathrm{x}}\right) \in \mathscr{C}_{k} \rightarrow \Delta(\mathrm{x})=\left(b_{\mathrm{x}}, a_{\mathrm{x}}, d_{\mathrm{x}}, c_{\mathrm{x}}\right) \in \mathscr{C}_{k},
$$

which implies:

$$
x_{\mathrm{p}_{n}}=y_{\mathrm{q}_{n}}, y_{\mathrm{p}_{n}}=x_{\mathrm{q}_{n}}, s_{\mathrm{p}_{n}}=s_{\mathrm{q}_{n}}^{-1} .
$$

Finally, this class of representations reduce to the superintegrable chiral Potts model under the following special homogeneous limits:

$$
x_{\mathrm{q}_{n}}^{p} \rightarrow\left(1+k^{\prime}\right) / k, \quad \forall n \in\{1, \ldots, \mathrm{N}\} .
$$

\section{A.4 Reduction to the XXZ spin $s$ open chains at the $p=2 s+1$ roots of unit}

Here we show that imposing a set of conditions on the parameters of the $\tau_{2}$-Lax operator we can reduce it to the one of the spin $s=(p-1) / 2 \mathrm{XXZ}$ case at the $p$ roots of unit. This has the interesting consequence that the analysis done of the open $\tau_{2}$-chain reduces for these special representations to that of an open spin chain under the same boundary conditions. In particular, we have that our functional equation characterization of the spectrum under a special homogeneous limit defines the spectrum of the following local Hamiltonian given by fusion and the Sklyanin formula:

$$
\mathscr{H}_{s}=c_{0} \frac{d}{d \lambda} \mathscr{T}_{p}(\lambda)_{\lambda=q^{s}}+\text { constant }
$$

with

$$
\mathscr{H}_{s}=\sum_{n=1}^{\mathrm{N}-1} H_{n, n+1}^{(s)}+\frac{d}{d \lambda} K_{1,-}^{(p)}\left(q^{s}\right)+\frac{\operatorname{tr}_{0}\left\{K_{0,+}^{(p)}\left(q^{s}\right) H_{0, \mathrm{~N}}^{(s)}\right\}}{\operatorname{tr}_{0}\left\{K_{0,+}^{(p)}\left(q^{s}\right)\right\}},
$$

where $\mathscr{T}_{p}(\lambda)$ is the $p$-fused open transfer matrix, $H_{n, n+1}^{(s)}$ is the two sites local Hamiltonian of the spin $s$ XXZ chain, $K_{0, \pm}^{(p)}(\lambda)$ are the $p \times p$ matrices $p$-fused scalar solutions of the reflection algebra obtained by doing the fusion $p-1$ times starting from the original $2 \times 2$ scalar solutions $K_{0, \pm}(\lambda)$, respectively.

Lemma A.3. Let us fix the parameters of the $\tau_{2}$-representations as follows:

$$
\begin{aligned}
& \alpha_{n}=\beta_{n}=1 / 2, a_{n}=q^{-1 / 2} / 2 i, \\
& b_{n}=i q^{1 / 2} / 2, c_{n}=q^{-1 / 2} / 2 i, d_{n}=i q^{1 / 2} / 2,
\end{aligned}
$$

and let

$$
L_{a, n}^{X X Z}(\lambda)=\left(\begin{array}{cc}
{\left[\lambda q^{s+S_{n}^{z} / 2}-1 /\left(\lambda q^{s+S_{n}^{z} / 2}\right)\right] / 2} & S_{n}^{-} \\
S_{n}^{+} & {\left[\lambda q^{s-S_{n}^{z} / 2}-1 /\left(\lambda q^{s-S_{n}^{z} / 2}\right)\right] / 2}
\end{array}\right)
$$

be the Lax operator of the spin $s X X Z$ chain with anisotropy $\cosh \eta=(q+1 / q) / 2$, then it holds:

$$
L_{a, n}^{X X Z}(\lambda)=L_{a, n}(\lambda / q)
$$


for $s=(p-1) / 2$ and $q=e^{i \pi p^{\prime} / p}$ with $p^{\prime}$ even and $p$ odd and coprime, which is equivalent to the following identities among the generators of the local algebras:

$$
S_{n}^{+}=u_{n}^{-1}\left(v_{n}-1 / v_{n}\right) / 2 i, \quad S_{n}^{-}=u_{n}\left(v_{n} / q-q / v_{n}\right) / 2 i
$$

and

$$
S_{n}^{z}=\frac{2 p}{i \pi p^{\prime}} \log v_{n}-(p+1) \in\{-2 s,-2(s-1), \ldots, 2 s\} \bmod 2 p
$$

Proof. Let us denote

$$
\overline{|a, n\rangle}=\left(\begin{array}{lllll}
0 & \cdots & 1 & \cdots & 0
\end{array}\right)^{t_{0}}, \quad a \in\{1, \ldots, 2 s+1\}
$$

the element $a$ of the canonical basis given by the column vector with all elements zero except that in row $a$ which is 1 . In this basis we have the following representations for the generators

$$
S_{n}^{+}=\left(\begin{array}{cccc}
0 & f(1) & & \\
& \ddots & & \\
& & \ddots & f(2 s) \\
& & & 0
\end{array}\right), S_{n}^{-}=\left(\begin{array}{cccc}
0 & & & \\
f(1) & \ddots & & \\
& \ddots & \ddots & \\
& & f(2 s) & 0
\end{array}\right) \text {, }
$$

where:

$$
f(j)=\sqrt{\sinh j \eta \sinh (p-j) \eta}=i\left(q^{j}-q^{-j}\right) / 2,
$$

and the second identity holds for $q^{p}=1$, and:

$$
S_{n}^{z}=\left(\begin{array}{cccc}
2 s & 0 & & \\
0 & \ddots & \ddots & \\
& \ddots & \ddots & 0 \\
& & 0 & -2 s
\end{array}\right)
$$

Let us now impose that in our representation the $v_{n}$-eigenstates coincide with the elements of the canonical basis:

$$
|p-a, n\rangle=\overline{|a+1, n\rangle} \forall a \in\{0, \ldots, p-1\},
$$

we can verify now the formulae (A.52)-(A.53). The formula (A.53) is equivalent to:

$$
v_{n}=q^{\left(S_{n}^{z}+p+1\right) / 2}
$$

which holds for the following identities:

$$
q^{\left(S_{n}^{z}+p+1\right) / 2} \overline{|a+1, n\rangle}=\overline{|a+1, n\rangle} q^{(2(s-a)+p+1) / 2}=|p-a, n\rangle q^{p-a}=v_{n}|p-a, n\rangle .
$$

Similarly we have:

$$
\begin{aligned}
S_{n}^{+} \overline{|a, n\rangle} & =\overline{|a+1, n\rangle} f(a)=\overline{|a+1, n\rangle}\left(q^{-a}-q^{a}\right) / 2 i \\
& =\left[u_{n}^{-1}\left(v_{n}-1 / v_{n}\right) / 2 i\right]|p-a, n\rangle \quad \forall a \in\{1, \ldots, p\}
\end{aligned}
$$

and

$$
\begin{aligned}
S_{n}^{-} \overline{|a, n\rangle} & =\overline{|a-1, n\rangle} f(a-1)=\overline{|a-1, n\rangle}\left(q^{1-a}-q^{a-1}\right) / 2 i \\
& =\left[u_{n}\left(v_{n} / q-q / v_{n}\right) / 2 i\right]|p+2-a, n\rangle \quad \forall a \in\{1, \ldots, p\} .
\end{aligned}
$$




\section{References}

[1] M. Gaudin, Boundary Energy of a Bose Gas in One Dimension, Phys. Rev. A 4, 386 (1971), doi:10.1103/PhysRevA.4.386.

[2] F. C. Alcaraz, M. N. Barber, M. T. Batchelor, R. J. Baxter and G. R. W. Quispel, Surface exponents of the quantum XXZ, Ashkin-Teller and Potts models, J. Phys. A 20, 6397 (1987), doi:10.1088/0305-4470/20/18/038.

[3] E. K. Sklyanin, Boundary conditions for integrable quantum systems, J. Phys. A 21, 2375 (1988), doi:10.1088/0305-4470/21/10/015.

[4] I. V. Cherednik, Factorizing particles on a half-line and root systems, Theor. Math. Phys. 61, 977 (1984), doi:10.1007/BF01038545.

[5] P. P. Kulish and E. K. Sklyanin, The general Uq(sl(2)) invariant XXZ integrable quantum spin chain, J. Phys. A 24, L435 (1991), doi:10.1088/0305-4470/24/8/009.

[6] L. Mezincescu, R. I. Nepomechie and V. Rittenenberg, Bethe ansatz solution of the FateevZamolodchikov quantum spin chain with boundary terms, Phys. Lett. A 147, 70 (1990), doi:10.1016/0375-9601(90)90016-H.

[7] L. Mezincescu and R. Nepomechie, Integrability of open spin chains with quantum algebra symmetry, Int. J. Mod. Phys. A 6, 5231 (1991), doi:10.1142/S0217751X9200257X.

[8] V. Pasquier and H. Saleur, Common structures between finite systems and conformal field theories through quantum groups, Nucl. Phys. B 330, 523 (1990), doi:10.1016/05503213(90)90122-T.

[9] M.T. Batchelor, L. Mezincescu, R.I. Nepomechie and V. Rittenberg, q-deformations of the O(3) symmetric spin-1 Heisenberg chain, J. Phys. A 23, L141 (1990), doi:10.1088/03054470/23/4/003.

[10] P. P. Kulish and E. K. Sklyanin, Algebraic structures related to the reflection equations, J. Phys. A 25(22), 5963 (1992), doi:10.1088/0305-4470/25/22/022.

[11] H. J. de Vega and A. González-Ruiz, The Highest Weight property for the SUq(n) invariant spin chains, J. Phys. A 26, L519 (1993), doi:10.1016/0370-2693(94)90868-0.

[12] H. J. de Vega and A. Gonzalez-Ruiz, Boundary K-matrices for the XYZ, XXZ and XXX spin chains, J. Phys. A 27, 6129 (1994), doi:10.1088/0305-4470/27/18/021.

[13] S. Ghoshal and A. B. Zamolodchikov, Boundary S-matrix and boundary state in twodimensional integrable quantum field theory, Int. J. Mod. Phys. A 9, 3841 (1994), doi:10.1142/S0217751X94001552;

S. Ghoshal and A. B. Zamolodchikov, Errata: Boundary S-matrix and boundary state in two-dimensional integrable quantum field theory, Int. J. Mod. Phys. A 9, 4353 (1994) doi:10.1142/S0217751X94002430.

[14] M. Jimbo, R. Kedem, T. Kojima, H. Konno and T. Miwa, XXZ chain with a boundary, Nucl. Phys. B 441, 437 (1995), doi:10.1016/0550-3213(95)00062-W.

[15] M. Jimbo, R. Kedem, H. Konno, T. Miwa, and R. Weston, Difference equations in spin chains with a boundary, Nucl. Phys. B 448, 429 (1995), doi:10.1016/05503213(95)00218-H. 
[16] N. Kitanine, K. K. Kozlowski, J. M. Maillet, G. Niccoli, N. A. Slavnov and V. Terras, Correlation functions of the open XXZ chain: I, J. Stat. Mech. P10009 (2007), doi:10.1088/1742-5468/2007/10/P10009;

N. Kitanine, K. K. Kozlowski, J. M. Maillet, G. Niccoli, N. A. Slavnov and V. Terras, Correlation functions of the open XXZ chain: II J. Stat. Mech. P07010 (2008), doi:10.1088/1742-5468/2008/07/P07010.

[17] A. Doikou, Fused integrable lattice models with quantum impurities and open boundaries, Nucl. Phys. B 668, 447 (2003), doi:10.1016/j.nuclphysb.2003.07.001.

[18] L. Frappat, R. Nepomechie and E. Ragoucy, A complete Bethe ansatz solution for the open spin-s XXZ chain with general integrable boundary terms, J. Stat. Mech. P09009 (2007), doi:10.1088/1742-5468/2007/09/P09009.

[19] N. Crampé, E. Ragoucy and D. Simon Eigenvectors of open XXZ and ASEP models for a class of non-diagonal boundary conditions, J. Stat. Mech. P11038 (2010), doi:10.1088/1742-5468/2010/11/P11038.

[20] N. Crampé and E. Ragoucy, Generalized coordinate Bethe ansatz for non-diagonal boundaries, Nucl. Phys. B 858, 502 (2012), doi:10.1016/j.nuclphysb.2012.01.020.

[21] P. Baseilhac and K. Koizumi, A deformed analogue of Onsager's symmetry in the XXZ open spin chain, J. Stat. Mech. P10005 (2005), doi:10.1088/1742-5468/2005/10/P10005; P. Baseilhac and K. Koizumi, Exact spectrum of the XXZ open spin chain from the q-Onsager algebra representation theory, J. Stat. Mech. P09006 (2007), doi:10.1088/17425468/2007/09/P09006.

[22] R. I. Nepomechie, Solving the open XXZ spin chain with non-diagonal boundary terms at roots of unity, Nucl. Phys. B 622, 615 (2002), doi:10.1016/S0550-3213(01)00585-5;

R. I. Nepomechie, Functional relations and Bethe Ansatz for the XXZ chain, J. Stat. Phys. 111, 1363 (2003), doi:10.1023/A:1023016602955.

[23] R. I. Nepomechie and F. Ravanini, Completeness of the Bethe Ansatz solution of the open XXZ chain with non-diagonal boundary terms, J. Phys. A36, 11391 (2003), doi:10.1088/0305-4470/36/45/003.

[24] R. Murgan, R. I. Nepomechie, and C. Shi, Exact solution of the open XXZ chain with general integrable boundary terms at roots of unity, J. Stat. Mech. P08006 (2006), doi:10.1088/1742-5468/2006/08/P08006.

[25] W. Galleas, Functional relations from the Yang-Baxter algebra: Eigenvalues of the XXZ model with non-diagonal twisted and open boundary conditions, Nucl. Phys. B 790, 524 (2008), doi:10.1016/j.nuclphysb.2007.09.011.

[26] W.-L Yang, R. I. Nepomechie and Y.-Z. Zhang, Q-operator and T-Q relation from the fusion hierarchy, Phys. Lett. B 633, 664 (2006), doi:10.1016/j.physletb.2005.12.022.

[27] J. Cao, W.-L. Yang, K. Shi and Y. Wang, Off-diagonal Bethe ansatz solutions of the anisotropic spin-1/2 chains with arbitrary boundary fields, Nucl. Phys. B 877, 152 (2013), doi:10.1016/j.nuclphysb.2013.10.001.

[28] D. E. Derkachov, G. P. Korchemsky and A. N. Manashov, Baxter Q-operator and Separation of Variables for the open SL(2,R) spin chain, J. High Energy Phys. 0310, 053 (2003), doi:10.1088/1126-6708/2003/10/053. 
[29] H. Frahm, A. Seel, T. Wirth, Separation of Variables in the open XXX chain, Nucl. Phys. B 802, 351 (2008), doi:10.1016/j.nuclphysb.2008.04.008.

[30] H. Frahm, J. H. Grelik A. Seel and T. Wirth, Functional Bethe ansatz methods for the open XXX chain, J. Phys. A 44, 015001 (2011), doi:10.1088/1751-8113/44/1/015001.

[31] L. Amico, H. Frahm, A. Osterloh, and T. Wirth, Separation of variables for integrable spinboson models, Nucl. Phys. B 839, 604 (2010), doi:10.1016/j.nuclphysb.2010.07.005.

[32] L. Amico, H. Frahm, A. Osterloh and G. A. P. Ribeiro, Integrable spin-boson models descending from rational six-vertex models, Nucl. Phys. B 787, 283 (2007), doi:10.1016/j.nuclphysb.2007.07.022.

[33] G. Niccoli, Non-diagonal open spin-1/2 XX Z quantum chains by separation of variables: complete spectrum and matrix elements of some quasi-local operators, J. Stat. Mech. P10025 (2012), doi:10.1088/1742-5468/2012/10/P10025.

[34] S. Faldella and G. Niccoli, SOV approach for integrable quantum models associated with general representations on spin-1/2 chains of the 8-vertex reflection algebra, J. Phys. A 47(11), 115202 (2014), doi:10.1088/1751-8113/47/11/115202.

[35] S. Faldella, N. Kitanine and G. Niccoli, The complete spectrum and scalar products for the open spin-1/2 XXZ quantum chains with non-diagonal boundary terms, J. Stat Mech. P01011 (2014), doi:10.1088/1742-5468/2014/01/P01011.

[36] N. Kitanine, J. M. Maillet, G. Niccoli, Open spin chains with generic integrable boundaries: Baxter equation and Bethe ansatz completeness from separation of variables, J. Stat. Mech. P05015 (2014), doi:10.1088/1742-5468/2014/05/P05015.

[37] H. Fan, B.-Y. Hou, K.-J. Shi and Z.-X. Yang, Algebraic Bethe ansatz for eight vertex model with general open-boundary conditions, Nucl. Phys. B 478, 723 (1996), doi:10.1016/0550-3213(96)00398-7.

[38] J. Cao, H.-Q. Lin, K.-J. Shi and Y. Wang, Exact solution of XXZ spin chain with unparallel boundary fields, Nucl. Phys. B 663, 487 (2003), doi:10.1016/S0550-3213(03)00372-9.

[39] W.-L. Yang and Y.-Z. Zhang, On the second reference state and complete eigenstates of the open XXZ chain, J. High Energy Phys. 4, 044 (2007), doi:10.1088/1126$6708 / 2007 / 04 / 044$.

[40] D. Arnaudon, N. Crampé, A. Doikou, L. Frappat and E. Ragoucy, Analytical Bethe Ansatz for closed and open gl(n)-spin chains in any representation, J. Stat. Mech. P02007 (2005), doi:10.1088/1742-5468/2005/02/P02007;

D. Arnaudon, N. Crampé, A. Doikou, L. Frappat and E. Ragoucy, Spectrum and Bethe ansatz equations for the $U_{q}(g l(N))$ closed and open spin chains in any representation An. H. Poincaré 7, 1217 (2006), doi:10.1007/s00023-006-0280-x.

[41] E. Ragoucy and G. Satta, Analytical Bethe Ansatz for closed and open gl(M|N) super-spin chains in arbitrary representations and for any Dynkin diagram, J. High Energy Phys. 9, 001 (2007), doi:10.1088/1126-6708/2007/09/001.

[42] S.Belliard and E. Ragoucy, The nested Bethe ansatz for 'all' open spin chains with diagonal boundary conditions, J. Phys. A 42, 205203 (2009), doi:10.1088/17518113/42/20/205203. 
[43] H. Schulz, Hubbard chain with reflecting ends, J. Phys. C 18, 581 (1985), doi:10.1088/0022-3719/18/3/010.

[44] H. Q. Zhou, Quantum integrability for the one-dimensional Hubbard open-chain, Phys. Rev. B 54, 41 (1996), doi:10.1103/PhysRevB.54.41;

H. Q. Zhou, Graded reflection equations and the one-dimensional Hubbard open chain Phys. Lett. A 228, 48 (1997), doi:10.1016/S0375-9601(97)00035-2.

[45] H. Asakawa and M. Suzuki, Finite-size corrections in the XXZ model and the Hubbard model with boundary fields, J. Phys. A 29, 225 (1996), doi:10.1088/03054470/29/2/004.

[46] X.W. Guan, M. S. Wang and S. D. Yang, Lax pair and boundary K-matrices for the one-dimensional Hubbard model, Nucl. Phys. B 485, 685 (1997), doi:10.1016/S05503213(96)00630-X.

[47] X. W. Guan, Algebraic Bethe ansatz for the one-dimensional Hubbard model with open boundaries, J. Phys. A 33, 5391 (2000), doi:10.1088/0305-4470/33/30/309.

[48] M. Shiroishi and M. Wadati, Bethe Ansatz equation for the Hubbard model with boundary fields, J. Phys. Soc. Japan 66, 1 (1997), doi:10.1143/JPSJ.66.1;

M. Shiroishi and M. Wadati, Integrable boundary conditions for the one-dimensional Hubbard model J. Phys. Soc. Japan 66, 2288 (1997), doi:10.1143/JPSJ.66.2288.

[49] F. C. Alcaraz, M. Droz, M. Henkel and V. Rittenberg, Reaction-Diffusion Processes, Critical Dynamics, and Quantum Chains, Ann. Phys. 230, 250 (1994), doi:10.1006/aphy.1994.1026.

[50] Z. Bajnok, Equivalences between spin models induced by defects, J. Stat. Mech. P06010 (2006), doi:10.1088/1742-5468/2006/06/P06010.

[51] J. de Gier and F. H. L. Essler, Bethe Ansatz Solution of the Asymmetric Exclusion Process with Open Boundaries Phys. Rev. Lett. 95, 240601 (2005), doi:10.1103/PhysRevLett.95.240601;

J. de Gier and F. H. L. Essler, Exact spectral gaps of the asymmetric exclusion process with open boundaries, J. Stat. Mech. P12011 (2006), doi:10.1088/17425468/2006/12/P12011.

[52] A. Doikou, J. Stat. Mech. P09010 (2006), doi:10.1088/1742-5468/2006/09/P09010.

[53] B. Derrida, An exactly soluble non-equilibrium system: the asymmetric simple exclusion process, Phys. Rep. 301, 65 (1998), doi:10.1016/S0370-1573(98)00006-4.

[54] G. M. Schütz, in Phase Transitions and Critical Phenomena 9, edited by C. Domb and J. L. Lebowitz, London: Academic (2000).

[55] J. Sirker, R. G. Pereira, and I. Affleck, Diffusion and Ballistic Transport in One-Dimensional Quantum Systems, Phys. Rev. Lett. 103, 216602 (2009), doi:10.1103/PhysRevLett.103.216602.

[56] T. Prosen, Open XXZ Spin Chain: Nonequilibrium Steady State and a Strict Bound on Ballistic Transport Phys. Rev. Lett. 106, 217206 (2011), doi:10.1103/PhysRevLett.106.217206.

[57] P. Roche and D. Arnaudon, Irreducible representations of the quantum analogue of SU(2), Lett. Math. Phys. 17, 295-300 (1989), doi:10.1007/BF00399753. 
[58] E. Date, M. Jimbo, K. Miki and T. Miwa, $R$ matrix for cyclic representations of $U_{q}(s l(3, C))$ at $q^{3}=1$, Phys. Lett. A 148, 45 (1990), doi:10.1016/0375-9601(90)90573-7.

[59] E. Date, M. Jimbo, K. Miki and T. Miwa, Generalized chiral Potts models and minimal cyclic representations of $U_{q}(g l(n, C))$, Comm. Math. Phys. 137, 133 (1991), doi:10.1007/978-1-4612-0315-5_14.

[60] V. Tarasov, Cyclic Monodromy Matrices for the R-matrix of the Six-Vertex Model and the Chiral Potts Model with Fixed Spin Boundary Conditions, Int. J. Mod. Phys. A 7, 963-975 (1992), doi:10.1142/S0217751X92004129;

A. Tsuchia, T. Eguchi and M. Jimbo (eds.), RIMS Research Project 1992 infinite Analysis. Proceedings, Singapore, World Scientific (1992).

[61] V. Tarasov, Cyclic monodromy matrices for sl(n) trigonometric R-matrices, Comm. Math. Phys. 158, 459-483 (1993), doi:10.1007/BF02096799.

[62] E. K. Sklyanin and L. D. Faddeev, Quantum mechanical approach to completely integrable field theory models, Sov. Phys. Dokl. 23, 902 (1978).

[63] E. K. Sklyanin and L. A. Takhtajan and L. D. Faddeev, Quantum inverse problem method I, Theor. Math. Phys. 40, 688 (1980), doi:10.1007/BF01018718.

[64] P. P. Kulish, N. Y. Reshetikhin and E. K. Sklyanin, Yang-Baxter equation and representation theory: I Lett. Math. Phys. 5, 393 (1981), doi:10.1007/BF02285311.

[65] A. N. Kirillov and N. Y. Reshetikhin, Exact solution of the integrable XXZ Heisenberg model with arbitrary spin. II. Thermodynamics of the system, J. Phys. A 20, 1565 (1987), doi:10.1088/0305-4470/20/6/039.

[66] N. Yu Reshetikhin, A Method Of Functional Equations In The Theory Of Exactly Solvable Quantum Systems, Lett. Math. Phys. 7205 (1983), doi:10.1007/BF00400435;

N. Yu Reshetikhin The functional equation method in the theory of exactly soluble quantum systems, Sov. Phys. JETP, 691 (1983).

[67] V. V. Bazhanov and N. Y. Reshetikhin, Critical RSOS models and conformal field theory, Int. J. Mod. Phys. A 4, 115 (1989), doi:10.1142/S0217751X89000042.

[68] L. A. Takhtajan and L. D. Faddeev, The quantum method of the inverse problem and the Heisenberg XYZ model, Russ. Math. Surv. 34(5), 11 (1979), doi:10.1070/RM1979v034n05ABEH003909.

[69] E. K. Sklyanin, Inverse scattering method and quantum nonlinear Schrödinger equation, Dokl. Akad. Nauk SSSR 244, 1337 (1979);

E. K. Sklyanin, Method of the inverse scattering problem and the nonlinear quantum Schrödinger equation, Sov. Phys. Dokl. 24, 107 (1979).

[70] P. P. Kulish and E. K. Sklyanin, it Quantum inverse scattering method and the Heisenberg ferromagnet, Phys. Lett. A 70, 461 (1979), doi:10.1016/0375-9601(79)90365-7.

[71] L. D. Faddeev, Quantum completely integral models of field theory, Sov. Sci. Rev. Math. Cl 107 (1980).

[72] E. K. Sklyanin, Quantum version of the method of inverse scattering problem, J. Sov. Math. 19, 1546 (1982), doi:10.1007/BF01091462.

[73] L. D. Faddeev, Les Houches lectures of 1982, Elsevier Sci, Publ. 563 (1984). 
[74] L. D. Faddeev, How Algebraic Bethe Ansatz works for integrable model, Les-Houches lectures, arXiv:hep-th/9605187.

[75] M. Jimbo, Yang-Baxter equation in integrable systems, Adv. Series in Math. Phys. 10, Singapore, World Scientific, (1990).

[76] P. P. Kulish and E. K. Sklyanin, Quantum spectral transform method: recent developments, Lect. Notes in Phys. 151, 61 (1982), doi:10.1007/3-540-11190-5_8.

[77] B. S. Shastry, S. S. Jha and V. Singh, Exactly Solvable Problems in Condensed Matter and Relativistic Field Theory, Lect. Notes in Phys. 242 (1985), doi:10.1002/zamm.19870670606.

[78] H. B. Thacker, Exact integrability in quantum field theory and statistical systems, Rev. Mod. Phys. 53, 253 (1981), doi:10.1103/RevModPhys.53.253.

[79] A. G. Izergin and V. E. Korepin, Lattice version of quantum field theory models in two dimensions, Nucl. Phys. B 205, 401 (1982), doi:10.1016/0550-3213(82)90365-0.

[80] H. Bethe, Zur Theorie der Metalle. I. Eigenwerte und Eigenfunktionen der linearen Atomkette., Z. Phys. 71, 205 (1931), doi:10.1007/BF01341708.

[81] R. J. Baxter, Exactly Solved Models in Statistical Mechanics, Academic Press, New York, U.S.A. (1982).

[82] E. H. Lieb and D. C. Mattis, Mathematical Physics in One Dimension, New-York: Academic (1966).

[83] M. T. Batchelor, R. J. Baxter, M. J. O'Rourke and C. M. Yung, Exact solution and interfacial tension of the six-vertex model with anti-periodic boundary conditions, J. Phys. A 28, 2759 (1995), doi:10.1088/0305-4470/28/10/009.

[84] E. K. Sklyanin, The quantum Toda chain, Lect. Notes Phys. 226, 196 (1985), doi:10.1007/3-540-15213-X_80.

[85] E. K. Sklyanin, Goryachev-Chaplygin top and the inverse scattering method, J. Sov. Math. 31, 3417 (1985), doi:10.1007/BF02107243.

[86] E.K. Sklyanin, Quantum inverse scattering method. Selected topics, in: Quantum groups and quantum integrable systems, World Scientific, 63 (1992).

[87] E. K. Sklyanin, Separation of variables, new trends, Prog. Theor. Phys. Suppl. 118, 35 (1995), doi:10.1143/PTPS.118.35.

[88] O. Babelon, D. Bernard and F. Smirnov, Quantization of solitons and the restricted sineGordon model, Comm. Math. Phys. 182, 319 (1996), doi:10.1007/BF02517893;

O. Babelon, D. Bernard and F. Smirnov, Null-vectors in integrable field theory, Comm. Math. Phys. 186, 601 (1997), doi:10.1007/s002200050122.

[89] F. Smirnov, Structure of matrix elements in the quantum Toda chain, J. Phys. A 31, 8953 (1998), doi:10.1088/0305-4470/31/44/019.

[90] S. E. Derkachov, G. P. Korchemsky and A. N. Manashov, Separation of variables for the quantum SL(2,R) spin chain, J. High Energy Phys. 07, 047 (2003), doi:10.1088/11266708/2003/07/047. 
[91] A. Bytsko and J. Teschner, Quantization of models with non-compact quantum group symmetry. Modular XXZ magnet and lattice sinh-Gordon model, J. Phys. A 39, 12927 (2006), doi:10.1088/0305-4470/39/41/S11.

[92] G. von Gehlen, N. Iorgov, S. Pakuliak and V. Shadura, The Baxter-Bazhanov-Stroganov model: separation of variables and the Baxter equation, J. Phys. A 39, 7257 (2006), doi:10.1088/0305-4470/39/23/006;

G. von Gehlen, N. Iorgov, S. Pakuliak and V. Shadura, Factorized finite-size Ising model spin matrix elements from separation of variables, J. Phys. A 42, 304026 (2009), doi:10.1088/1751-8113/42/30/304026.

[93] G. von Gehlen, N. Iorgov, S. Pakuliak, V. Shadura and Y. Tykhyy, Form-factors in the Baxter-Bazhanov-Stroganov model I: norms and matrix elements, J. Phys. A 40, 14117 (2007), doi:10.1088/1751-8113/40/47/006;

G. von Gehlen, N. Iorgov, S. Pakuliak, V. Shadura and Y. Tykhyy, Form-factors in the Baxter-Bazhanov-Stroganov model II: Ising model on the finite lattice, J. Phys. A 41, 095003 (2008), doi:10.1088/1751-8113/41/9/095003.

[94] G. Niccoli and J. Teschner, The sine-Gordon model revisited: I, J. Stat. Mech. P09014 (2010), doi:10.1088/1742-5468/2010/09/P09014.

[95] G. Niccoli, Reconstruction of Baxter Q-operator from Sklyanin SOV for cyclic representations of integrable quantum models, Nucl. Phys. B 835, 263 (2010), doi:10.1016/j.nuclphysb.2010.03.009.

[96] N. Grosjean and G. Niccoli, The $\tau_{2}$-model and the chiral Potts model revisited: Completeness of Bethe equations originated from Sklyanin SOV, J. Stat. Mech. P11005 (2012), doi:10.1088/1742-5468/2012/11/P11005

[97] N. Grosjean, J. M. Maillet, G. Niccoli, On form factors of local operators in the lattice sine-Gordon model, J. Stat. Mech. P10006 (2012), doi:10.1088/17425468/2012/10/P10006;

N. Grosjean, J. M. Maillet, G. Niccoli, On the Form Factors of Local Operators in the Bazhanov-Stroganov and Chiral Potts Models, An. H. Poincaré 16, 1103 (2015), doi:10.1007/s00023-014-0358-9.

[98] S. Niekamp, T. Wirth and H. Frahm, The XXZ model with anti-periodic twisted boundary conditions, J. Phys. A 42, 195008 (2009), doi:10.1088/1751-8113/42/19/195008.

[99] G. Niccoli, Antiperiodic spin-1/2 XXZ quantum chains by separation of variables: Form factors and complete spectrum, Nucl. Phys. B 870, 397 (2013), doi:10.1016/j.nuclphysb.2013.01.017;

G. Niccoli, On the developments of Sklyanin's quantum separation of variables for integrable quantum field theories, arXiv:1301.4924.

[100] G. Niccoli, Form factors and complete spectrum of XXX antiperiodic higher spin chains by quantum separation of variables, J. Math. Phys. 54, 053516 (2013), doi:10.1063/1.4807078;

G. Niccoli, An antiperiodic dynamical six-vertex model: I. Complete spectrum by SOV, matrix elements of the identity on separate states and connections to the periodic eight-vertex model J. Phys. A 46(7), 075003 (2013), doi:10.1088/1751-8113/46/7/075003.

[101] G. Niccoli and V. Terras, The 8-vertex model with quasi-periodic boundary conditions, J. Phys. A 49, 044001 (2015), doi:10.1088/1751-8113/49/4/044001. 
[102] G. Niccoli and V. Terras, Antiperiodic XXZ chains with arbitrary spins: Complete eigenstate construction by functional equations in separation of variables, Lett. Math. Phys. 105, 989 (2015), doi:10.1007/s11005-015-0759-9.

[103] D. Levy-Bencheton, G. Niccoli and V. Terras, Antiperiodic dynamical 6-vertex model by separation of variables II: Functional equations and form factors, J. Stat. Mech. 033110 (2016), doi:10.1088/1742-5468/2016/03/033110.

[104] N. Kitanine, J.M. Maillet, G. Niccoli and V. Terras, On determinant representations of scalar products and form factors in the SoV approach: the XXX case, J. Phys. A 49, 104002 (2016), doi:10.1088/1751-8113/49/10/104002.

[105] N. Kitanine, J.M. Maillet, G. Niccoli and V. Terras, The open XXX spin chain in the SoV framework: scalar product of separate states, arXiv:1606.06917.

[106] W. Heisenberg, Zur Theorie des Ferromagnetismus, Z. Physik 49, 619 (1928), doi:10.1007/BF01328601;

L. Hulthén, Über das Austauschproblem eines Kristalles, Ark. Mat. Astron. Fys. 26, 1 (1938);

R. Orbach, Linear Antiferromagnetic Chain with Anisotropic Coupling Phys. Rev. 112, 309 (1958), doi:10.1103/PhysRev.112.309;

L. R. Walker, Antiferromagnetic Linear Chain, Phys. Rev. 116, 1089 (1959), doi:10.1103/PhysRev.116.1089.

[107] C. N. Yang and C. P. Yang, One-Dimensional Chain of Anisotropic Spin-Spin Interactions. I. Proof of Bethe's Hypothesis for Ground State in a Finite System, Phys. Rev. 150, 321 (1966), doi:10.1103/PhysRev.150.321;

C. N. Yang and C. P. Yang, One-Dimensional Chain of Anisotropic Spin-Spin Interactions. II. Properties of the Ground-State Energy Per Lattice Site for an Infinite System Phys. Rev. 150, 327 (1966), doi:10.1103/PhysRev.150.327.

[108] M. Gaudin, La Fonction d'onde de Bethe. Paris, Masson (1983).

[109] B. S. Shastry, Infinite Conservation Laws in the One-Dimensional Hubbard Model Phys. Rev. Lett. 56, 1529 (1986), doi:10.1103/PhysRevLett.56.1529;

B. S. Shastry, Decorated star-triangle relations and exact integrability of the one dimensional Hubbard model, J. Stat. Phys. 30, 57 (1987), doi:10.1007/BF01022987.

[110] E. Olmedilla, M. Wadati and Y. Akutsu, Yang-Baxter Relations for Spin Models and Fermion Models, J. Phys. Soc. Japan. 56, 2298 (1987), doi:10.1143/JPSJ.56.2298.

[111] E. Olmedilla and M. Wadati, Conserved quantities of the one-dimensional Hubbard model, Phys. Rev. Lett. 60, 1595 (1988), doi:10.1103/PhysRevLett.60.1595.

[112] M. J. Martins and P. B. Ramos, Exact solution of the lattice vertex model analogue of the coupled Bariev XY chains, J. Phys. A 30, L465 (1997), doi:10.1088/03054470/30/14/004;

M. J. Martins and P.B. Ramos, The quantum inverse scattering method for Hubbard-like models Nucl. Phys. B 522, 413 (1998), doi:10.1016/S0550-3213(98)00199-0.

[113] V. V. Bazhanov and Yu. G. Stroganov, Chiral Potts model as a descendant of the six vertex model, J. Stat. Phys. 59, 799 (1990), doi:10.1007/BF01025851.

[114] N. Kitanine, J. M. Maillet, and V. Terras, Form factors of the XXZ Heisenberg spin-1/2 finite chain, Nucl. Phys. B 554, 647 (1999), doi:10.1016/S0550-3213(99)00295-3. 
[115] J. M. Maillet and V. Terras, On the quantum inverse scattering problem, Nucl. Phys. B 575, 627 (2000), doi:10.1016/S0550-3213(00)00097-3.

[116] R. J. Baxter, Eight-vertex model in lattice statistics and one-dimensional anisotropic Heisenberg chain. I. Some fundamental eigenvectors, Ann. Phys. 76, 1 (1973), doi:10.1016/0003-4916(73)90439-9;

R. J. Baxter, Eight-vertex model in lattice statistics and one-dimensional anisotropic Heisenberg chain. II. Equivalence to a generalized ice-type model, Ann. Phys. 76, 25 (1973), doi:10.1016/0003-4916(73)90440-5;

R. J. Baxter, Eight-vertex model in lattice statistics and one-dimensional anisotropic Heisenberg chain. III. Eigenvectors of the transfer matrix and Hamiltonian, Ann. Phys. 76, 48 (1973), doi:10.1016/0003-4916(73)90441-7.

[117] H. Au-Yang and J. H. H. Perk, Eigenvectors in the superintegrable model I: $s l_{2}$ generators, Phys. A 41, 275201 (2008), doi:10.1088/1751-8113/41/27/275201;

$\mathrm{H}$. Au-Yang and J. H. H. Perk, Eigenvectors in the superintegrable model II: ground-state sector, J. Phys. A 42, 375208 (2009), doi:10.1088/1751-8113/42/37/375208.

[118] R. J. Baxter, V. V. Bazhanov and J. H. H. Perk, Functional relations for transfer matrices of the chiral Potts model, Int. J. Mod. Phys. B 4, 803 (1990), doi:10.1142/S0217979290000395.

[119] R. J. Baxter, Superintegrable chiral Potts model : Thermodynamic properties, an "inverse" model, and a simple associated Hamiltonian, J. Stat. Phys. 57, 1 (1989), doi:10.1007/BF01023632;

R. J. Baxter, The superintegrable chiral Potts model Phys. Lett. A 133, 185 (1989), doi:10.1016/0375-9601(88)91014-6.

[120] G. Albertini, B. M. McCoy and J. H. H. Perk, Eigenvalue Spectrum of the Superintegrable Chiral Potts Model, Adv. Study in Pure Math. 19, 1 (1989);

G. Albertini, B. M. McCoy and J. H. H. Perk, Commensurate-incommensurate transition in the ground state of the superintegrable chiral Potts model, Phys. Lett. A 135, 159 (1989), doi:10.1016/0375-9601(89)90254-5;

G. Albertini, B. M. McCoy and J. H. H. Perk, Level crossing transitions and the massless phases of the superintegrable chiral Potts chain, Phys. Lett. A 139, 204 (1989), doi:10.1016/0375-9601(89)90142-4.

[121] R. J. Baxter, J. H. H. Perk and H. Au-Yang, New solutions of the star-triangle relations for the chiral Potts model, Phys. Lett. A 128, 138 (1988), doi:10.1016/03759601(88)90896-1;

H. Au-Yang and J. H. H. Perk, Onsager's star triangle equation: Master key to the integrability, Adv. Studies in Pure Math. 19 Kinokuniya: Academic (1989).

[122] H. Au-Yang, B. M. McCoy, J. H. H. Perk, S. Tang, and M. Yan, Commuting transfer matrices in the chiral Potts models: Solutions of star-triangle equations with genus $>1$, Phys. Lett. A 123, 219 (1987), doi:10.1016/0375-9601(87)90065-X.

[123] B. M. McCoy, J. H. H. Perk, S. Tang and C. H. Sah, Commuting transfer matrices for the 4 state self-dual chiral Potts model with a genus 3 uniformizing Fermat curve, Phys. Lett. A 125, 9 (1987), doi:10.1016/0375-9601(87)90509-3.

[124] H. Au-Yang, B. M. McCoy, J. H. H. Perk and S. Tang, Solvable models in statistichal mechanics and Riemann surfaces of genus greater than one, Algebraic Analysis 1, M. Kashiwara and T. Kawai (eds.), Academic Press, New York (1988). 
[125] V. O. Tarasov, Transfer matrix of the superintegrable chiral Potts model. Bethe ansatz spectrum, Phys. Lett. A 147, 487 (1990), doi:10.1016/0375-9601(90)90612-R.

[126] R. J. Baxter, Transfer Matrix Functional Relations for the Generalized $\tau_{2}\left(t_{q}\right)$ Model, J. Stat. Phys. 117, 1 (2004), doi:10.1023/B:JOSS.0000044062.64287.b9. 